\title{
Consensus consumer and intertemporal asset pricing with heterogeneous beliefs*
}

\author{
Elyès Jouini, \\ Institut universitaire de France, \\ IFD and CEREMADE, Université Paris Dauphine \\ Clotilde Napp, \\ CNRS, DRM-Université Paris Dauphine and CREST
}

December 18, 2006

*We wish to thank Jean-Michel Grandmont, Hedi Kallal and José Scheinkman for useful comments on earlier drafts as well as two anonymous referees and the Associate Editor. We have benefited from comments from seminar and conference participants at the Institut Henri Poincaré (Bachelier Seminar), London Business School, Princeton (Civitas Foundation Finance Seminar), MIT, French Finance Association Conference (Lyon), Blaise Pascal Conference on Financial Modelling (Paris), Universita della Svizzera Italiana (Lugano), Bocconi University (Milan), University of British Columbia (Vancouver) and in particular Harjoat Bhamra, Markus Brunnermeier and Dimitri Vayanos. 


\begin{abstract}
The aim of the paper is to analyze the impact of heterogeneous beliefs in an otherwise standard competitive complete market economy. The construction of a consensus probability belief, as well as a consensus consumer, are shown to be valid modulo an aggregation bias, which takes the form of a discount factor. In classical cases, the consensus probability belief is a risk tolerance weighted average of the individual beliefs, and the discount factor is proportional to beliefs dispersion. This discount factor makes the heterogeneous beliefs setting fundamentally different from the homogeneous beliefs setting, and it is consistent with the interpretation of beliefs heterogeneity as a source of risk.

We then use our construction to rewrite in a simple way the equilibrium characteristics (market price of risk, risk premium, risk-free rate) in a heterogeneous beliefs framework and to analyze the impact of beliefs heterogeneity. Finally, we show that it is possible to construct specific parametrizations of the heterogeneous beliefs model that lead to globally higher risk premia and lower risk-free rates.
\end{abstract}

JEL numbers: G10, G12, D84

Keywords : risk premium, beliefs heterogeneity, optimism, pessimism, consensus consumer 


\section{Introduction}

The representative agent approach introduced by Negishi (1960) and developed by Rubinstein (1974), Breeden and Litzenberger (1978), Constantinides (1982) has become a significant cornerstone of theoretical and applied macroeconomics and has been the basis for many developments in finance. Among these developments, the Capital Asset Pricing Model (CAPM, Sharpe 1964 and Lintner 1965) and the Consumption based CAPM (CCAPM, Ingersoll, 1987, Huang and Litzenberger, 1988, Duffie, 1996) play an important role. Given their empirical tractability, these models have generated extensive empirical tests and subsequent theoretical extensions. However, as mentioned by Williams (1977), "difficulties remain, significant among which is the restrictive assumption of homogeneous expectations". It has been since repeatedly argued that the diversity of investors forecasts is an important part of any proper understanding of the workings of asset markets ${ }^{1}$.

The aim of the present paper is to analyze the consequences of the introduction of heterogeneous subjective beliefs in an otherwise standard Arrow-Debreu equilibrium economy. More precisely, we start from a given equilibrium with heterogeneous beliefs in an otherwise standard complete market model and, as in Calvet et al. (2002) in a static setting, we investigate the following issues: 1) Is it possible to define a consensus belief, i.e. a belief which, if held by all individuals, would generate the same equilibrium prices and trade volumes as in the actual heterogeneous economy? 2) Is it still possible in such a context to define a representative agent (or consensus consumer)? 3) What is the impact of beliefs heterogeneity on the risk premium (or market price of risk)? 4) What is the impact of beliefs heterogeneity on the risk-free rate?

5) What is the impact of beliefs heterogeneity on the assets price?

In this paper, we take the different subjective beliefs as given. As in Varian (1985, 1989), Abel (1989) or Harris-Raviv (1993), they reflect difference of opinion among the agents rather

\footnotetext{
${ }^{1}$ See e.g. Lintner (1969), Rubinstein (1975, 1976), Gonedes (1976), Miller (1977), Williams (1977), Jarrow (1980), Mayshar (1981, 1983), Cragg-Malkiel (1982), Varian (1985, 1989), Abel (1989), Harris-Raviv (1993), Detemple-Murthy (1994), Basak (2000), Gallmeyer (2000), Welch (2000), Ziegler (2000), Gallmeyer-Hollifield (2002), Calvet et al. (2002), Diether et al. (2002).
} 
than difference of information; indeed, "we assume that investors receive common information, but differ in the way they interpret this information" (Harris-Raviv, 1993). The different subjective beliefs might come from a Bayesian updating of the investors predictive distribution over the uncertain returns on risky securities as in e.g. Williams (1977), Detemple-Murthy (1994), Zapatero (1998), Gallmeyer (2000), Basak (2000), Gallmeyer-Hollifield (2002), but we do not make such an assumption. Notice that the above mentioned models with learning are not "more endogeneous" since the investor updating rule and the corresponding probabilities can be determined separately from his/her optimization problem (see e.g. Genotte, 1986).

The paper is organized as follows. We present in Section 2 a method to aggregate in an intertemporal framework heterogeneous individual subjective beliefs into a single consensus belief. Given an observed equilibrium with heterogeneous probabilities, we look for a consensus belief, which, if held by all investors would lead to an equivalent equilibrium, in the sense that it would leave invariant trading volumes and equilibrium market prices. The aim of this construction is to analyze whether from the econometrician point of view and without access to microdata (i.e. data on individual behaviors) there is a way to distinguish between homogeneous beliefs and heterogeneous beliefs equilibria.

We start by proving the existence of a consensus characteristic. However, this characteristic fails to be a proper belief in the sense that its density is not a martingale (or, in other words, does not satisfy the law of iterated expectations) and, as a result, it can not be directly interpreted as a change of probability. In fact, it is possible to decompose this consensus characteristic into a proper consensus belief (a true probability belief) and a discount factor. We find then that equilibrium prices and trading volumes in a heterogeneous beliefs economy are the same as in an equivalent homogeneous beliefs economy in which agents would share the same consensus probability belief, which is an average of the individual beliefs, but would discount their utility from future consumption. As a consequence of the presence of this discount effect, the heterogeneous beliefs setting cannot in general be simply reduced to an homogeneous beliefs setting, with an average belief. 
It is then easy to construct a consensus consumer who, when endowed with the total wealth in the economy, generates the same equilibrium prices as in the original equilibrium. The belief of this representative agent is the previously found consensus probability belief, his/her utility function is the same as in the standard setting, but the representative agent has in general a different discount factor than the representative consumer that can be constructed in the usual case of common beliefs. For example, when all agents have the same discount factor, the consensus consumer typically has a different (non constant) discount factor.

This discount factor is (at least in the classical HARA utility functions setting) proportional to beliefs dispersion and might be positive or negative depending on whether the investor is "cautious" or not. A possible interpretation of this discount consists in considering the dispersion of beliefs as a source of risk. Indeed, when there is more risk involved, depending on whether the investor is "cautious" or not, he/she will reduce or increase current consumption with respect to future consumption (precautionary savings), acting as if his/her utility was discounted by a negative or positive discount rate. In the heterogeneous beliefs framework, investors act as if they were in an homogeneous beliefs setting with an additional source of risk and this effect is captured, in the aggregation procedure, by the time-varying discount factor.

Concerning the consensus probability belief, it appears as a weighted average of the original individual probability beliefs, and the weights are given, at least in the HARA setting, by the individual risk tolerances. More risk tolerant agents see their beliefs better represented in the consensus belief. This is a natural result. Indeed, if an agent is fully insured by other agents, it is intuitive that this agent's belief should not affect the equilibrium prices. Only those who bear a share of the risk should see their expectations be taken into account at the aggregate level.

When the utility functions are logarithmic, the consensus probability belief is a wealthweighted average of the individual beliefs as first found by Rubinstein (1976). Notice however that in this case the discount factor turns to be a constant, equal to one, which makes the logarithmic utility functions case very specific.

We give credit to Calvet et al. (2002) for the idea of constructing a representative agent in 
the heterogeneous beliefs setting modulo the introduction of some degree of freedom and their paper actually is the starting point of our work. However our construction is very different and so is the rest of our analysis. Our degree of freedom is given by the introduction of a discount factor instead of an adjustment of aggregate wealth as in Calvet et al. $(2002)^{2}$.

In Section 3, we analyze the impact of beliefs heterogeneity on the market price of risk, the risk-free rate and asset prices. For this purpose we consider a continuous-time framework, where risk is generated by a Brownian motion. We adopt this framework in order to compare our results with the standard ones obtained, among others, by Merton (1973), Breeden and Litzenberger (1978), Breeden (1979) and Duffie and Zame (1989). The choice of Itô processes makes it possible to focus on situations with a finite number of sources of risk and hence a finite number of possible sources of disagreement (note that, in an Itô processes framework, all agents necessarily agree on the assets volatility). For the sake of simplicity, all results are presented in a one-dimensional Brownian motion setting but, as underlined in footnote 26, they can be easily generalized to a multi-dimensional setting.

We derive an adjusted CCAPM formula. We prove that only the change of probability (from the initial one to the consensus one) has an impact on the market price of risk. We find that the CCAPM formula with heterogeneous beliefs is given by the CCAPM formula in an economy where all investors would share the same probability belief namely the probability consensus belief obtained through the aggregation procedure. The impact of heterogeneous beliefs on the market price of risk is then very clear: it leads to an increase (resp. decrease) of the market price of risk (with respect to the homogeneous setting) if and only if the consensus probability is pessimistic (resp. optimistic), where pessimistic is meant in the sense that the instantaneous aggregate wealth growth rate (under this probability) is lower that under the objective initial

\footnotetext{
${ }^{2}$ Note that Calvet et al. results are in a static setting and that the extension of their results to a dynamic setting requires the introduction of a rescaling time varying stochastic (predictable) process and not only a scalar adjustment. A discount factor seems more natural, from an economic point of view, than a rescaling of the aggregate endowment. In our approach, utility functions are aggregated into a representative utility function, beliefs are aggregated into a representative belief and time discount factors are aggregated into a representative time discount factor. Besides, the aggregation procedure of Calvet et al. and its generalization to a dynamic setting do not permit to disentangle the construction of the consensus probability and of the rescaling process and leads to risk-premium and risk-free rate formulas that are hard to interpret.
} 
probability. In fact, the equity premium subjectively expected is the same for pessimistic and optimistic agents and the reason why pessimism increases the objective expectation of the equity premium is not that a pessimistic representative agent requires a higher risk premium. He/She requires the same equity premium but his/her pessimism leads him/her to underestimate the average rate of return of equity (leaving unchanged his/her estimation of the risk-free rate). Thus the objective expectation of the equilibrium premium is greater than the representative agent's subjective expectation, hence is greater than the standard equity premium. Our results are consistent with those of Abel (2002), Cechetti et al. (2000), Hansen et al. (1999), or Anderson et al. (2000), which introduce distorded beliefs associated to cautious/pessimistic individual behavior, but the main difference lies in the fact that in our framework, optimism/pessimism is relevant at the aggregate level and not at the individual one. In particular, it is possible to have optimism/pessimism at the aggregate level, even in models where the average (equal-weighted) belief is neutral (neither optimistic, nor pessimistic).

By contrast with the market price of risk, both the change of probability and the discount factor have an impact on the risk-free rate. The impact of the change of probability contributes to a lowering of the risk free rate if and only if the consensus probability is pessimistic. Indeed, if the representative agent is pessimistic about the growth rate of aggregate wealth, then relative to the standard case, he/she will attempt to reduce current consumption and increase current savings. The attempt to increase current savings puts then downward pressure on the interest rate. Besides, the impact of the discount factor contributes to an increase (resp. decrease) of the risk free rate when the "discount rate" is nonnegative (resp. nonpositive), which has a clear interpretation: a nonnegative "discount rate" means that future consumption is less important for the representative agent, and leads to a higher equilibrium interest rate. For linear risk tolerance utility functions and for small beliefs dispersion, the first effect is quantitatively much more important than the second one.

Section 4 explores this further by giving explicit formulas and numerical results in the case of exponential or power utilities. In particular, we show that a positive correlation between wealth 
and optimism would induce an optimistic bias and a lower expected risk premium. Even though short sale contraints are not part of our model, under the positive correlation hypothesis we obtain that prices reflect the more optimistic view, as in e.g. Miller (1977). This would imply that assets with a higher beliefs dispersion should yield lower returns which is consistent with the findings of Diether et al. (2002), who "provide evidence that stocks with higher dispersion in analysts earnings forecasts earn lower future returns than otherwise similar stocks". Conversely a negative correlation between wealth and optimism would induce a pessimistic bias and a higher risk premium. Moreover, we show numerically, that if investors are on average pessimistic the risk premium is higher and the risk-free rate is lower than in the standard setting, which is interesting in light of the equity premium and the risk-free rate puzzles (Mehra-Prescott, 1985, and Weil, 1989).

Section 5 concludes. All the proofs are in the Appendix.

\section{Consensus belief, consensus consumer}

In the classical representative agent approach, all investors are taken as having the same subjective beliefs and the same opportunity sets. In this section, we analyze to what extent this approach can be extended to heterogeneous subjective beliefs. More precisely, we start from a given equilibrium with heterogeneous beliefs in an otherwise standard complete market model, and we explore to what extent it is possible 1) to define a consensus belief, i.e. a belief, which, if held by all individuals would generate the same equilibrium prices and trading volumes as in the actual heterogeneous economy and 2) to define a representative agent (or a consensus consumer). The starting point of our aggregation procedure is the paper by Calvet et al. (2002).

The model is standard, except that we allow the agents to have distinct subjective probabilities. We fix a finite time horizon $T$ on which we are going to treat our problem. We consider a probability space $(\Omega, F, P)$ and a filtration $\left(F_{t}\right)_{t \in[0, T]}$ on $(\Omega, F)$ with $F_{T}=F$ which satisfies the usual conditions ${ }^{3}$. As usual, the interval $[0, T]$ is endowed with the Lebesgue measure

\footnotetext{
${ }^{3}$ i.e. $\left(F_{t}\right)_{t \in[0, T]}$ is right-continuous and $F_{0}$ contains all the $P$-negligible events in $F$.
} 
$\Lambda$ and we denote by $P \otimes \Lambda$ the product probability induced on the product space $\Omega \otimes[0, T]$. Each investor indexed by $i=1, \cdots, N$ solves a standard dynamic utility maximization problem. He/She has a current income at date $t$ denoted by $e_{t}^{*^{i}}$ and a von Neumann-Morgenstern utility function for consumption of the form $E^{Q^{i}}\left[\int_{0}^{T} u_{i}\left(t, c_{t}\right) d t\right]$, where $Q^{i}$ is a probability measure equivalent to $P$ which corresponds to the subjective belief of individual $i$. If we denote by $\left(M_{t}^{i}\right)_{t \in[0, T]}$ the positive density process of $Q^{i}$ with respect to $P$, then the utility function can be rewritten as $E^{P}\left[\int_{0}^{T} M_{t}^{i} u_{i}\left(t, c_{t}\right) d t\right]$. We denote by $e_{t}^{*}$ the aggregate current income at date $t$, i.e. $e_{t}^{*}=\sum_{i=1}^{n} e_{t}^{*^{i}}$.

We make the following classical assumptions.

\section{Assumption}

- $u_{i}:[0, T] \times\left[k_{i}, \infty\right) \rightarrow \mathbb{R} \cup\{-\infty\}$ is of class ${ }^{4} C^{1,1}$, increasing and strictly concave with respect to the second variable ${ }^{5}$,

- for $i=1, \cdots, N, P \otimes \Lambda\left\{e^{*^{i}}>k_{i}\right\}>0, P \otimes \Lambda\left\{e^{*^{i}} \geq k_{i}\right\}=1$ and $P \otimes \Lambda\left\{e^{*}>\sum_{i=1}^{N} k_{i}+\varepsilon\right\}=$ 1 for some $\varepsilon>0$,

- $E^{P}\left[\int_{0}^{T} e_{t}^{*} d t\right]<\infty$ and $P \otimes \Lambda\left\{M^{i}(t, \omega) \leq B\right\}=1$ for $i=1, \cdots, N$ and some $B>0$.

The first condition is a classical regularity condition. If we interpret $k_{i}$ as a minimum subsistence level, the second condition can be interpreted as a survival condition for each agent ${ }^{6}$ and for the whole economy. The last condition is technical and ensures that $E^{P}\left[\int_{0}^{T} M_{t}^{i} e_{t}^{*^{i}} d t\right]<$ $\infty$ hence, by concavity of $u_{i}$, that $E^{Q_{i}}\left[\int_{0}^{T} u_{i}\left(t, e_{t}^{*^{i}}\right) d t\right]<\infty^{7}$.

\footnotetext{
${ }^{4} \mathrm{~A}$ function $u(t, c)$ is said to be of class $C^{1, k}$ if it is continuously differentiable with respect to $t$ and $k$ times continuously differentiable with respect to $c$.

${ }^{5}$ Note that we could easily generalize all the following results to the case where $k_{i}$ is a function of $t$.

${ }^{6} \mathrm{In}$ fact, this condition can be weakened. For instance, if the equilibrium price is known, we only need to impose that

$$
E^{P}\left[\int_{0}^{T} q_{t}^{*} e_{t}^{*^{i}} d t\right]>E^{P}\left[\int_{0}^{T} q_{t}^{*} k_{i} d t\right]
$$

which permits, through trade, to reach allocations $\left(y^{*^{i}}\right)>k_{i} P \otimes \Lambda$ a.s.

${ }^{7}$ This condition can be replaced by $E^{P}\left[\int_{0}^{T}\left|e_{t}^{*^{i}}\right|^{p} d t\right]<\infty$ and $E^{P}\left[\int_{0}^{T}\left|M_{t}^{i}\right|^{q} d t\right]<\infty$ where $p$ and $q$ are such that $\frac{1}{p}+\frac{1}{q}=1$ which also ensure that $E^{P}\left[\int_{0}^{T} M_{t}^{i} e_{t}^{*^{i}} d t\right]<\infty$.
} 
We do not specify the utility functions $u_{i}$, although we shall focus on the classical cases of linear risk tolerance utility functions (which include logarithmic, power as well as exponential utility functions).

As underlined in the introduction, we take the different subjective probabilities as given. They might reflect difference of opinion among the agents. They might also come from a Bayesian updating of the investors predictive distribution over the uncertain returns on risky securities. Note that this does not necessarily lead to the same posterior for all agents as in Aumann (1976) because the agents might have different priors that are not necessarily based on information. They might also update their beliefs in a non Bayesian way, as in e.g. ScheinkmanXiong (2003) where some agents are overconfident.

In the remainder of the paper, an admissible consumption plan for agent $i$ is an adapted $\left[k_{i}, \infty\right)$-valued process $y^{i}$ such that $E^{P}\left[\int_{0}^{T}\left|y_{t}^{i}\right| d t\right]<\infty$. We recall that an Arrow-Debreu ${ }^{8}$ equilibrium relative to the beliefs $\left(M^{i}\right)$ and the income processes $\left(e^{*^{i}}\right)$ is defined by a positive price process $q^{*}$, in terms of date- 0 consumption units, and a family of optimal admissible consumption plans $\left(y^{*^{i}}\right)$ such that markets clear, i.e.

$$
\left\{\begin{array}{c}
y^{*^{i}}=y^{i}\left(q^{*}, M^{i}, e^{*^{i}}\right) \\
\sum_{i=1}^{N} y^{*^{i}}=\sum_{i=1}^{N} e^{*^{i}} \equiv e^{*}
\end{array}\right.
$$

where

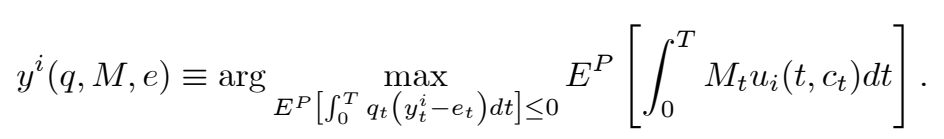

We start from an equilibrium $\left(q^{*},\left(y^{*^{i}}\right)\right)$ relative to the beliefs $\left(M^{i}\right)$ and the income processes $e^{*^{i}}$. Such an equilibrium, when it exists, can be characterized by the first order necessary conditions for individual optimality and the market clearing condition. These conditions

\footnotetext{
${ }^{8}$ We recall that Arrow-Debreu equilibria can be implemented, by continuous trading, in Radner equilibria with only a finite number of securities (see Duffie-Huang, 1985).
} 
can be written as follows

$$
\left\{\begin{array}{c}
M_{t}^{i} u_{i}^{\prime}\left(t, y_{t}^{*^{i}}\right) \leq \lambda_{i} q_{t}^{*}, \quad \text { on }\left\{y^{*^{i}}=k_{i}\right\} \\
M_{t}^{i} u_{i}^{\prime}\left(t, y_{t}^{*^{i}}\right)=\lambda_{i} q_{t}^{*}, \quad \text { on }\left\{y^{*^{i}}>k_{i}\right\} \\
E^{P}\left[\int_{0}^{T} q_{t}^{*}\left(y_{t}^{*^{i}}-e^{*^{i}}\right) d t\right]=0 \\
\sum_{i=1}^{N} y^{*^{i}}=e^{*}
\end{array}\right.
$$

for some set of positive Lagrange multipliers $\lambda=\left(\lambda_{i}\right)_{i=1, \cdots, N}$.

In the sequel, we will say that $\left(q^{*},\left(y^{*^{i}}\right)\right)$ is an interior equilibrium relative to the beliefs $\left(M^{i}\right)$ and the income processes $\left(e^{*^{i}}\right)$ if $^{9} y^{*^{i}}>k_{i}, P \otimes \Lambda$ a.s. for $i=1, \cdots, N$.

Our first aim is to find an "equivalent equilibrium" in which the heterogeneous subjective beliefs would be aggregated into a common characteristic $M$. Following the approach of Calvet et al. (2002), we shall define an "equivalent equilibrium" by two requirements. First, the "equivalent equilibrium" should generate the same equilibrium trading volumes $\left(y^{*^{i}}-e^{*^{i}}\right)$ and price process $q^{*}$ as in the original equilibrium with heterogeneous beliefs. Second, every investor should be indifferent at the margin between investing one additional unit of income in the original equilibrium with heterogeneous beliefs and in the "equivalent equilibrium", so that each asset gets the same marginal valuation by each investor (in terms of his/her marginal utility) in both equilibria ${ }^{10}$.

The existence of such an "equivalent equilibrium" is given by the following proposition.

Proposition 1. Consider an interior equilibrium $\left(q^{*},\left(y^{*^{i}}\right)\right)$ relative to the beliefs $\left(M^{i}\right)$ and the income processes $\left(e^{*^{i}}\right)$. There exists a unique positive and adapted process $\left(M_{t}\right)_{t \in[0, T]}$ with $M_{0}=1$, there exists a unique family of income processes $\left(\bar{e}^{i}\right)$ with $\sum_{i=1}^{N} \bar{e}^{i}=e^{*}$ and a unique family of individual consumption processes $\left(\bar{y}^{i}\right)$ such that $\left(q^{*},\left(\bar{y}^{i}\right)\right)$ is an equilibrium relative to the common characteristic $M$ and the income processes $\left(\bar{e}^{i}\right)$ and such that trading volumes and

\footnotetext{
${ }^{9}$ Note that under the following additional condition $u_{i}^{\prime}\left(t, k_{i}\right)=\infty$ for $t \in[0, T]$ and $i=1, \cdots, N$, all the equilibria are interior ones.

${ }^{10}$ This requirement is in fact equivalent to the condition that each investor's observed (or initial) demand be larger than (resp. equal to, less than) his/her demand in the "equivalent equilibrium" if and only if he/she attaches a subjective probability that is larger than (resp. equal to, less than) the aggregate common probability, which appears as a natural requirement for an aggregation procedure.
} 
individual marginal valuation remain the same, i.e.

$$
\begin{aligned}
y^{*^{i}}-e^{*^{i}} & =\bar{y}^{i}-\bar{e}^{i} \quad i=1, \cdots, N \\
M_{t}^{i} u_{i}^{\prime}\left(t, y^{*^{i}}\right) & =M_{t} u_{i}^{\prime}\left(t, \bar{y}^{i}\right), \quad t \in[0, T], i=1, \cdots, N .
\end{aligned}
$$

This means that $\left(q^{*},\left(\bar{y}^{i}\right)\right)$ is an equilibrium with income transfers relative to the common characteristic $M$ and the income processes $\left(e^{*^{i}}\right)$ such that individual marginal valuation is the same as in the original equilibrium with heterogeneous beliefs. In other words, we proved that the initial equilibrium price process and trading volumes are also equilibrium price process and trading volumes in an homogeneous beliefs setting with the same aggregate wealth (but a possible different allocation of this wealth across agents). The positive process $M$ can then be interpreted as a consensus characteristic. In particular, if there is no heterogeneity, i.e. if all investors have the same belief represented by $M^{i}=\tilde{M}$ for all $i$, we obtain $M=\tilde{M}, \bar{e}^{i}=e^{*^{i}}$ and $\bar{y}^{i}=y^{*^{i}}$ for all $i$.

The individual marginal valuation invariance property is in fact equivalent to the invariance of the Lagrange multipliers. The cost we paid in order to maintain the Lagrange multipliers invariant has been to authorize a modification of individual incomes. Since our second aim is to construct a representative agent that would represent the economy, this is not really a cost. Indeed, from the representative agent point of view only aggregate characteristics are meaningful and "fictitious" feasible income transfers between agents (i.e. $\sum_{i=1}^{N} \bar{e}^{i}=\sum_{i=1}^{N} e^{*^{i}}$ ) in the construction of the "equivalent equilibrium" might be authorized. In other words, we characterize equilibria that are compatible with at least a feasible distribution of the total wealth among agents.

It is now easy to construct as in the standard case, a representative agent, i.e. an expected utility maximizing aggregate investor, representing the economy in equilibrium. 
For a given equilibrium $\left(q^{*},\left(y^{*^{i}}\right)\right)$ relative to the beliefs $\left(M^{i}\right)$, we introduce the function

$$
u_{\lambda}(t, x)=\max _{\sum_{i=1}^{N} x_{i} \leq x} \sum_{i=1}^{N} \frac{1}{\lambda_{i}} u_{i}\left(t, x_{i}\right)
$$

where $(\lambda)$ is the set of Lagrange multipliers provided by Equation (2.1).

Proposition 2. Consider an interior equilibrium $\left(q^{*},\left(y^{*^{i}}\right)\right)$ relative to the beliefs $\left(M^{i}\right)$ and the income processes $\left(e^{*^{i}}\right)$. There exists a consensus investor defined by the normalized von Neumann-Morgenstern utility function $u_{\lambda}$ and the consensus characteristic $M$ of Proposition 1., in the sense that the portfolio $e^{*}$ maximizes his/her expected utility $E^{P}\left[\int_{0}^{T} M_{t} u_{\lambda}\left(t, c_{t}\right) d t\right]$ under the market budget constraint $E^{P}\left[\int_{0}^{T} q_{t}^{*}\left(c_{t}-e_{t}^{*}\right) d t\right] \leq 0$.

The construction of the representative agent is exactly the same as in the standard setting. As a consequence, all classical properties of the representative agent utility function remain valid in our setting (see e.g. Huang-Litzenberger, 1988). Among other properties, if all individual utility functions are state independent, then the aggregate utility function is also state independent ${ }^{11}$, and if all individual utility functions exhibit linear risk tolerance, i.e. are such that $T_{i}(t, x) \stackrel{\text { def }}{=}$ $-\frac{u_{i}^{\prime}(t, x)}{u_{i}^{\prime \prime}(t, x)}=\theta_{i}+\eta x$, where all derivatives are with respect to $x$, then the aggregate utility function is also such that $-\frac{u^{\prime}(t, x)}{u^{\prime \prime}(t, x)}=\bar{\theta}+\eta x$ where $\bar{\theta}=\sum_{i=1}^{N} \theta_{i}$.

Example 2.1. $\quad$ 1. If the individual utility functions are of exponential type, more precisely

$$
\begin{gathered}
\text { if } u_{i}^{\prime}(t, x)=a_{i} e^{-\rho_{i} t} e^{-x / \theta_{i}} \text {, then } u_{\lambda}^{\prime}(t, x)=a e^{-\bar{\rho} t} e^{-x / \bar{\theta}} \text { for } a=e^{e_{0}^{*} / \bar{\theta}}, \bar{\rho}=\sum_{i=1}^{N} \frac{\theta_{i}}{\bar{\theta}} \rho_{i} \text { and } \\
M=\prod_{i=1}^{N}\left(M^{i}\right)^{\theta_{i} / \bar{\theta}} .
\end{gathered}
$$

2. If the individual utility functions are of power type, more precisely if $u_{i}^{\prime}(t, x)=b_{i} e^{-\rho t}\left(\theta_{i}+\eta x\right)^{-\frac{1}{\eta}}$

\footnotetext{
${ }^{11}$ Remark that our aggregation procedure applies to a framework where agents have common beliefs but possibly different state dependent utility functions of the following "separable" form $U_{i}(t, \omega, x)=v_{i}(t, \omega) u_{i}(t, x)$. In that case, we obtain a representative agent utility function of the same form $U(t, \omega, x)=v(t, \omega) u(t, x)$, where $u$ is obtained from the $u_{i}$ 's as in the standard framework, and where $v$ is an average of the $v_{i}$ 's (note that even if the $v_{i}$ 's are not martingales, our results still apply).
} 


$$
\begin{aligned}
& \text { for } \eta \neq 0 \text {, then } u_{\lambda}^{\prime}(t, x)=b e^{-\rho t}(\bar{\theta}+\eta x)^{-\frac{1}{\eta}} \text { for } b=\left(\bar{\theta}+\eta e_{0}^{*}\right)^{\frac{1}{\eta}} \text { and } \\
& \qquad \begin{array}{c}
M=\left[\sum_{i=1}^{N} \gamma_{i}\left(M^{i}\right)^{\eta}\right]^{\frac{1}{\eta}} \\
=\left[\sum_{i=1}^{N} \tau_{i}\left(M^{i}\right)^{-\eta}\right]^{-\frac{1}{\eta}} \\
\text { for } \gamma_{i}=\frac{\left(b_{i} / \lambda_{i}\right)^{\eta}}{\sum_{j=1}^{N}\left(b_{j} / \lambda_{j}\right)^{\eta}} \text { and } \tau_{i}=\frac{T_{i}\left(t, y^{*^{i}}\right)}{\sum_{j=1}^{N} T_{j}\left(t, y^{* i}\right)}
\end{array}
\end{aligned}
$$

The consensus characteristic $M$ is a martingale (i.e. the density process of a given probability) only when $\eta=1$ (logarithmic case). It is a supermartingale when $\eta<1$, and a submartingale when $\eta>1$.

Notice that it is not possible (except in the exponential case) to construct $M$ and to obtain $q^{*}$ as functions of the aggregate characteristics (e.g. consumption) of the economy and not of the individual ones.

However, as we shall see in the next section, the formulation ${ }^{12} q_{t}^{*}=M_{t} u_{\lambda}^{\prime}\left(t, e_{t}^{*}\right)$ which is a direct consequence of Proposition 2, will enable us to compare equilibrium under heterogeneous beliefs with equilibrium in the standard setting.

It is interesting to notice in Example 2.1 that for all utility functions in the classical class of linear risk tolerance utility functions, the consensus characteristic is obtained as a risk tolerance weighted average of the individual subjective beliefs ${ }^{13}$. Intuitively, the collective attitude towards risk depends upon how risk is allocated among individuals. For example, if an agent is fully insured by other agents, it is intuitive that this agent's beliefs should not affect the equilibrium

\footnotetext{
${ }^{12}$ by construction, the representative agent's Lagrange multiplier is normalized to 1 .

${ }^{13}$ It is easy to see that in the general case, the consensus characteristic can still be considered as an average of the individual beliefs. Since for all $i$, we have $\frac{1}{\lambda_{i}} M_{t}^{i} u_{i}^{\prime}\left(t, y_{t}^{*^{i}}\right)=M u^{\prime}\left(t, e_{t}^{*}\right)$ we obtain

$$
e_{t}^{*}=\sum_{i=1}^{N} I_{u_{i}}\left(t, \lambda_{i} \frac{M_{t}}{M_{t}^{i}} u^{\prime}\left(t, e^{*}\right)\right)=\sum_{i=1}^{N} I_{u_{i}}\left(t, \frac{M_{t}}{M_{t}^{i}} u_{i}^{\prime}\left(t, \bar{y}_{t}^{i}\right)\right)
$$

It is clear then that we cannot have $M_{t}>M_{t}^{i}$, for all $i$, with a positive probability. Indeed, this would lead to $\sum_{i=1}^{N} I_{u_{i}}\left(t, \frac{M_{t}}{M_{t}^{i}} u_{i}^{\prime}\left(t, \bar{y}_{t}^{i}\right)\right)<\sum_{i=1}^{N} \bar{y}_{t}^{i}=e_{t}^{*}$ with a positive probability which contradicts the equations above. The case $M_{t}>M_{t}^{i}$ is treated symmetrically.
} 
price. Only those who bear a share of the risk should see their expectations be taken into account at the aggregate level.

The process $M$ represents a consensus characteristic; however, as seen above, except in the logarithmic case, it fails to be a martingale. Consequently, it cannot be interpreted as a proper belief, i.e. the density process of a given probability measure. It is easy to see that it is not possible in general to recover the consensus characteristic as a martingale, as soon as we want the equilibrium price to remain the same and the optimal allocations in the equivalent equilibrium to be feasible, in the sense that they still add up to $e^{*}$ (even if we do not impose the invariance of individual marginal valuation $)^{14}$.

This means that in the general case, there is a bias induced by the aggregation of the individual probabilities into a consensus probability. We shall see in the following propositions that the utility function of the representative agent is not an expectation of the future utility from consumption but an expectation of a discounted future utility from consumption. The (possibly negative) discount rate will be on average nonnegative (resp. zero, resp. nonpositive) when $M$ is a supermartingale (resp. martingale, resp. submartingale).

In order to further specify our model, let us assume that $\left(F_{t}\right)_{t \in[0, T]}$ is the $P$-augmentation of the natural filtration generated by a one-dimensional Brownian motion $W$ on $(\Omega, F, P)$, and that $e^{*}$ and $M^{i}, i=1, \cdots, N$, are positive Itô processes that satisfy the following stochastic differential representation

$$
\begin{aligned}
d e_{t}^{*} & =\bar{\alpha}(t) d t+\bar{\beta}(t) d W_{t}, \quad \beta>0, \\
d M_{t}^{i} & =\bar{\delta}^{i}(t) d W_{t}, \quad M_{0}^{i}=1 .
\end{aligned}
$$

\footnotetext{
${ }^{14}$ Let us consider again the exponential utility functions case $-\frac{u_{i}^{\prime}(t, x)}{u_{i}^{\prime \prime}(t, x)}=1$. Let us consider the "equivalent interior equilibrium" allocations $\left(\bar{y}_{t}^{i}\right)$ associated to the same equilibrium price $q^{*}$ as in the initial equilibrium and to a common belief $M$. The first order conditions then lead to $M_{t} u_{i}^{\prime}\left(t, \bar{y}_{t}^{i}\right)=\varsigma_{i} M_{t}^{i} u_{i}^{\prime}\left(t, y_{t}^{*^{i}}\right)$ where $M$ is the candidate consensus belief and where the $\varsigma_{i}$ 's are given positive multipliers. This leads to

$$
M_{t}=\left(\prod_{i=1}^{N} \varsigma_{i}\right)^{1 / N}\left(\prod_{i=1}^{N} M_{t}^{i}\right)^{1 / N}
$$
}

and $M$ is a martingale only if all the $M^{i}$ 's are equal. 
where $\bar{\alpha}, \bar{\beta}$ and $\bar{\delta}^{i}$, for $i=1, \cdots, N$ are $\left(F_{t}\right)_{t \in[0, T]}$-adapted stochastic processes ${ }^{15}$ such that $\int|\bar{\alpha}(t)| d t<\infty, \int \bar{\beta}^{2}(t) d t<\infty$ and $\int\left(\bar{\delta}^{i}\right)^{2}(t) d t<\infty$, for $i=1, \cdots, N$.

Let us denote by $\alpha, \beta$ and $\delta^{i}$, for $i=1, \cdots, N$, respectively $\frac{\bar{\alpha}}{e^{*}}, \frac{\bar{\beta}}{e^{*}}$ and $\frac{\bar{\delta}^{i}}{M^{i}}$.

If we further assume that the utility functions are of class $C^{1,3}$, the first order conditions for an interior equilibrium and Itô's Lemma give us that the equilibrium price $q^{*}$, the equilibrium allocations $\left(y^{*^{i}}\right)$ as well as $M$ also satisfy stochastic differential representations of the form

$$
\begin{aligned}
d q_{t}^{*} & =\mu_{q^{*}}(t) q_{t}^{*} d t+\sigma_{q^{*}}(t) q_{t}^{*} d W_{t} \\
d y_{t}^{*^{i}} & =\mu_{y^{*}}^{i}(t) y_{t}^{*^{i}} d t+\sigma_{y^{*}}^{i}(t) y_{t}^{*^{i}} d W_{t} \\
d M_{t} & =\mu^{M}(t) M_{t} d t+\delta^{M}(t) M_{t} d W_{t}
\end{aligned}
$$

All the coefficients are $\left(F_{t}\right)_{t \in[0, T]}$-adapted stochastic processes. In the next, we will assume that $\delta^{M}$ satisfies Novikov's condition ${ }^{16}$, i.e. $E\left[\exp \left(\frac{1}{2} \int_{0}^{T}\left(\delta_{t}^{M}\right)^{2} d t\right)\right]<\infty$.

Proposition 3. Consider an interior equilibrium price process $q^{*}$ relative to the beliefs $\left(M^{i}\right)$, and the income processes $\left(e^{*^{i}}\right)$. There exists a positive martingale process $\bar{M}$ with $\bar{M}_{0}=1$, and a finite variation positive process $B$ with $B_{0}=1$ such that, with the notations of the previous propositions

$$
\bar{M}_{t} B_{t} u^{\prime}\left(t, e_{t}^{*}\right)=q_{t}^{*} .
$$

These processes are given by

$$
\begin{aligned}
B_{t} & =\exp \int_{0}^{t} \mu^{M}(s) d s \\
\bar{M}_{t} & =\exp \left(\int_{0}^{t} \delta^{M}(s) d W_{s}-\frac{1}{2} \int_{0}^{t}\left(\delta^{M}(s)\right)^{2} d s\right) .
\end{aligned}
$$

The process $B$ measures the default of martingality of the consensus characteristic $M$ and

\footnotetext{
${ }^{15}$ There is no Markovian assumption; $\bar{\alpha}, \bar{\beta}$ and $\bar{\delta}^{i}$, for $i=1, \cdots, N$ may depend on the entire past history of the economy.

${ }^{16}$ This condition ensures that $\int \delta^{M} d W$ is a martingale. For linear risk tolerance utility functions, it is easy to check that this condition is in particular satisfied if, for $i=1, \cdots, N$, we have $E\left[\exp \left(\frac{1}{2} \sum_{i=1}^{N} \int\left(\delta_{t}^{i}\right)^{2} d t\right)\right]<\infty$.
} 
leads to a (possibly negative) discount of utility from future consumption through the "discount rate" $\left(-\mu^{M}\right)$. The adjustment process $B$ measures then the aggregation bias induced by the heterogeneity of individual beliefs.

There are mainly two cases where there is no such adjustment effect, i.e. $B$ is constant and equal to 1 :

- if all investors share the same belief. The consensus belief $\bar{M}$ is then equal to that common belief,

- if all the utility functions are logarithmic. This property makes the case of logarithmic utility functions (which is often considered in the literature, see e.g. Rubinstein, 1976, Detemple-Murthy, 1994, Zapatero, 1998) very specific.

When $B$ fails to be constant, it is a natural concern to determine whether it is greater or smaller than 1 , increasing or decreasing. This will allow us to analyze the nature of this aggregation bias and its impact on the equilibrium state price density. For linear risk tolerance utility functions, the processes $\bar{M}$ and $B$ can be computed.

Proposition 4. For linear risk tolerance utility functions, we have

$$
\delta^{M}=\sum_{i=1}^{N} \tau_{i} \delta^{i}=E^{\tau}[\delta]
$$

and

$$
\mu^{M}=\frac{1}{2}(\eta-1)\left[\sum_{i=1}^{N} \tau_{i}\left(\delta^{i}\right)^{2}-\left(\sum_{i=1}^{N} \tau_{i} \delta^{i}\right)^{2}\right]=\frac{1}{2}(\eta-1) \operatorname{Var}^{\tau}[\delta]
$$

where $E^{\tau}$ (resp. $V a r^{\tau}$ ) denote the expected value (resp. variance) of $\delta$ across agents with a weight $\tau_{i}$ for agent $i$.

Notice that $\mu^{M} \leq 0$ if and only if $\eta \leq 1$ (this encompasses the exponential case).

For this class of utility functions, $\delta^{M}$ is a risk tolerance weighted average of the individual $\delta^{i}$, s and $\mu^{M}$ is proportional to the variance of the $\delta^{i}$ s with respect to the same weights. It appears then that the equilibrium price corresponds to an equilibrium price in an equivalent economy 
where the individual beliefs are replaced by a risk tolerance-weighted average belief and where an additional effect is introduced in order to take the initial heterogeneity into account. This effect is measured by $B$ or equivalently by $\mu^{M}$ which is directly related to beliefs dispersion.

Furthermore, it is easy to see that $B$ is nondecreasing, greater than 1 (resp. nonincreasing, lower than 1) if $\eta \geq 1$ (resp. $\eta \leq 1$ ). The technical reason for this result is the following. Depending on whether $\alpha<1$ or $\alpha>1$, the function $x^{\alpha}$ is convex or concave. Hence the supermartingale result for $\eta<1$, the submartingale result for $\eta>1$ and the martingale result for $\eta=1$. In most asset pricing models relative risk aversion is assumed to be larger than unity, or $\eta<1$ in our notation, which leads to a positive discount rate $\left(-\mu^{M}\right)$ and a decreasing $B$. However, the parameter $\eta$ has also another interpretation as a cautiousness parameter. Indeed, as shown by Gollier and Kimball (1996), the condition that absolute prudence is larger than twice the absolute risk aversion, which is given by $\eta>1$ in our HARA setting ${ }^{17}$, characterizes agent's willingness to save when facing risky situations. When there is more risk involved, depending on whether the investor is "cautious" or not, that is to say, depending on whether $\eta>1$ or $\eta<1$, the investor reduces or increases current consumption with respect to future consumption. For instance, for $\eta>1$, the investor is cautious and decreases current consumption acting as if his/her utility was discounted by a negative discount rate. This seems then to be a desirable property. Now in our context with heterogeneous beliefs, a possible interpretation consists in considering the dispersion of beliefs as a source of risk. More risk tolerant agents will insure less risk tolerant ones and will postpone their consumption. This is why at the collective level, the discount rate will overweight the views of individuals that are more risk tolerant.

Conversely, the introduction of the stochastic discount factor $B$ (at the individual level) in a homogeneous beliefs setting induces shocks on the utility functions and leads the agents to hedge these shocks. By doing this they mimic the behavior of the agents in the heterogeneous beliefs economy. These shocks are then the counterpart of beliefs heterogeneity and this is why

\footnotetext{
${ }^{17}$ Unfortunately HARA type functions do not permit to distinguish risk aversion from elasticity of intertemporal substitution. In a more general setting, it is likely that the condition for the discount rate $\left(-\mu^{M}\right)$ to be positive or negative would have hinged on the level of cautiousness, not on the level of risk aversion.
} 
the effect of beliefs heterogeneity might be compared to the effect of a new source of risk and why the heterogeneous beliefs setting is fundamentally different from the homogeneous beliefs setting.

To summarize, we have pointed out through previous propositions two distinct effects of the introduction of some beliefs heterogeneity on the equilibrium price .

There is first a change of probability effect from $P$ to the new common probability $Q$, whose density is given by $\bar{M}$. This aggregate probability $Q$ can be seen (at least for classical utility functions) as a weighted average of the individual subjective probabilities. The weights of this average are given by the individual risk tolerances exactly as in Rubinstein (1976) or DetempleMurthy (1994) where the authors focused on logarithmic utility functions.

The second effect is represented by an "aggregation bias" which is of finite variation and takes the form of a discount factor. We are able, for linear risk tolerance utility functions, to determine if it is associated to a positive or negative discount rate. Moreover, the adjustment process can be seen (at least in classical cases) as a measure of dispersion of individual beliefs.

We shall now analyze the impact of these two features on the equilibrium properties.

\section{Asset pricing with heterogeneous beliefs}

In this section, we use our construction of a representative consumer (Section 2) to study the impact of heterogeneity of beliefs on asset pricing.

We have obtained the following expression for the equilibrium (state) price (density) $q_{t}^{*}=$ $\overline{M_{t}} B_{t} u_{\lambda}^{\prime}\left(t, e_{t}^{*}\right)$ in the heterogeneous beliefs setting which we want to compare to the expression obtained in the standard setting, which is given by $q_{t}=u_{\lambda}^{\prime}\left(t, e_{t}^{*}\right)$. We consider as the standard setting an equilibrium under homogeneous beliefs (given by the objective probability $P$ ), for which the representative agent utility function is given by $u_{\lambda}$ with the same $\left(\lambda_{i}\right)$ as in our heterogeneous beliefs setting ${ }^{18}$.

\footnotetext{
${ }^{18}$ This is in particular the case when the standard setting equilibrium has the same Lagrange multipliers (or equivalently the same marginal valuations) as in our framework, or when investors have linear risk tolerance utility functions, since in that case (see e.g. Huang-Litzenberger, 1988), the representative agent utility function
} 


\subsection{Adjusted CCAPM, Market Price of Risk and Risk-free Rate}

We suppose the existence of a riskless asset with price process $S^{0}$ such that $d S_{t}^{0}=r^{f}(t) S_{t}^{0} d t$ and of a risky asset with price process ${ }^{19}$

$$
d S_{t}=S_{t} \mu^{R}(t) d t+S_{t} \sigma^{R}(t) d W_{t} \quad \sigma^{R}>0
$$

Through the use of Itô's formula, we easily obtain the following expression for the market price of risk $\left(\frac{\mu^{R}-r^{f}}{\sigma^{R}}\right)$ and the risk-free rate in the heterogeneous beliefs setting.

Proposition 5. The market price of risk (or Sharpe ratio) and the risk-free rate with heterogeneous beliefs are given by

$$
\begin{aligned}
M P R_{t}[\text { heterogeneous }] & =-\frac{u^{\prime \prime}\left(t, e_{t}^{*}\right)}{u^{\prime}\left(t, e_{t}^{*}\right)} \beta_{t} e_{t}^{*}-\delta_{t}^{M}=M P R_{t}[\text { standard }]-\delta_{t}^{M} \\
& =M P R_{t}[\text { homogeneous under } Q]
\end{aligned}
$$

and

$$
\begin{aligned}
r_{t}^{f}[\text { heterogeneous }] & =-\frac{u^{\prime \prime}\left(t, e_{t}^{*}\right)}{u^{\prime}\left(t, e_{t}^{*}\right)} \alpha_{t} e_{t}^{*}-\frac{1}{2} \frac{u^{\prime \prime \prime}\left(t, e_{t}^{*}\right)}{u^{\prime}\left(t, e_{t}^{*}\right)} \beta_{t}^{2} e_{t}^{* 2}-\mu_{t}^{M}-\delta_{t}^{M} \frac{u^{\prime \prime}\left(t, e_{t}^{*}\right)}{u^{\prime}\left(t, e_{t}^{*}\right)} \beta_{t} e_{t}^{*}(3 \\
& =r_{t}^{f}[\text { standard }]-\mu_{t}^{M}+\delta^{M}\left(-\frac{u^{\prime \prime}\left(t, e_{t}^{*}\right)}{u^{\prime}\left(t, e_{t}^{*}\right)} \beta_{t} e_{t}^{*}\right) \\
& =r_{t}^{f}[\text { homogeneous under } Q]-\mu_{t}^{M}
\end{aligned}
$$

Equation (3.1) is the CCAPM formula with heterogeneous beliefs. This adjusted formula differs from the classical one only through the change of probability from $P$ to the consensus probability $Q$ and the MPR in the heterogeneous beliefs setting is given by the MPR in an economy where all investors would share the same belief $Q$. The adjustment process $B$ plays no

does not depend upon the individual Lagrange multipliers (or initial allocations).

${ }^{19}$ We assume that the coefficients of these stochastic differential represenations satisfy the same conditions as in footnote 14 
role.

Beliefs heterogeneity increases the market price of risk if and only if $\delta^{M} \leq 0$. A nonpositive (resp. nonnegative) $\delta^{M}$ corresponds to a pessimistic (resp. optimistic) consensus probability. Indeed, letting $W_{t}^{Q}=W_{t}-\int_{0}^{t} \delta_{s}^{M} d s$, we know by Girsanov's Theorem that $W_{t}^{Q}$ is a $Q$-Brownian motion, and the dynamics of aggregate wealth $e^{*}$ under $Q$ is given by $d e_{t}^{*}=\left[\alpha_{t}+\delta_{t}^{M} \beta_{t}\right] e_{t}^{*} d t+\beta_{t} e_{t}^{*} d W_{t}^{Q}$, hence a nonpositive $\delta^{M}$ decreases the instantaneous aggregate wealth growth rate. Notice that a pessimistic consensus probability will also systematically decrease the instantaneous rate of return of any asset that is positively correlated with aggregate wealth. We have then obtained that optimism (resp. pessimism) at the consensus level decreases (resp. increases) the MPR. In fact, the MPR subjectively expected is not modified by the introduction of some beliefs dispersion or of some pessimism. In other words, the reason why pessimism increases the objective expectation of the MPR is not that pessimistic consumers require a higher risk premium. A pessimistic representative agent requires the same MPR but his/her pessimism leads him/her to underestimate the average rate of return of equity (leaving unchanged his/her estimation of the risk-free rate). Thus the objective expectation of the MPR is greater than the representative agent's subjective expectation, hence is greater than the standard MPR.

These results are consistent with those of Abel (2002), Cechetti et al. (2000), Epstein and Wang (1994), Hansen et al. (1999), or Anderson et al. (2000), which introduce distorded beliefs associated to cautious/pessimistic individual behavior. Since we have obtained that the MPR in the heterogeneous beliefs setting is in fact given by the MPR under the homogeneous belief $Q$, we are actually led to face the same issue as in e.g. Abel (2002), where investors all have the same subjective probability, different from the initial objective one ${ }^{20}$.

Unlike in Abel's paper setting, there is no need, in our setting, for all investors to be pessimistic. Pessimism at the aggregate level is sufficient in order to ensure an increase in the market

\footnotetext{
${ }^{20}$ Abel (2002) shows in a discrete time setting, and for power utility functions, that "uniform pessimism" (defined as a leftward translation of the objective distribution of the aggregate consumption) on the agents' (common) subjective probability leads to a higher risk premium.
} 
price of risk. More precisely, in the case of linear risk tolerance utility functions, there is no need for all $\delta^{i}$ to be nonpositive, it suffices that some average of the $\delta^{i}$, namely $\delta^{M}=\sum_{i=1}^{N} \tau_{i} \delta^{i}$, be nonpositive. We shall in Section 4 explore conditions on the individual beliefs under which the consensus probability is pessimistic (resp. optimistic).

By contrast with the MPR, both the change of probability effect and the discount factor have an impact on the risk-free rate. The impact of the discount factor is represented by $\mu^{M}$. If $B$ is nondecreasing (resp. nonincreasing), then $\mu^{M}$ is nonnegative (resp. nonpositive) and contributes to a decrease (resp. increase) of the risk-free rate. This effect has a clear interpretation; considering $\left(-\mu^{M}\right)$ as a discount rate, a nonpositive $\mu^{M}$ means that future consumption is less important for the representative agent, and leads to a higher equilibrium interest rate. We have seen that for power utility functions with $\eta<1$ (which is the reasonable case), as well as for exponential utility functions, $\mu^{M}$ is nonpositive, so that the effect of the aggregation bias is towards an increase of the interest rate which is bad news in light of the risk-free rate puzzle. For power utility functions with $\eta \geq 1$, we obtain ${ }^{21}$ a nonpositive discount rate $\left(-\mu^{M}\right)$ and as underlined by Weil (1989), "a value of $\beta$ above 1 (which corresponds in our setting to a nonpositive discount rate) is a computer's solution of the risk-free rate puzzle" ${ }^{22}$.

The impact of the change of probability from $P$ to the consensus probability $Q$ is represented by the covariance $\delta_{t}^{M}\left(-\frac{u^{\prime \prime}\left(t, e_{t}^{*}\right)}{u^{\prime}\left(t, e_{t}^{*}\right)} \beta_{t} e_{t}^{*}\right)$ and contributes to a lowering of the risk-free rate if and only if $Q$ is pessimistic, i.e. $\delta^{M} \leq 0$. The interpretation is the following: if consumers are pessimistic about the growth rate of aggregate wealth, then relative to the standard case, they will attempt to reduce current consumption and increase current savings. The attempt to increase current savings puts downward pressure on the interest rate.

Combining both effects, we obtain that the impact of heterogeneity of investors' beliefs is towards a lower (resp. higher) risk-free rate if the aggregate probability is pessimistic (resp. optimistic) and $B$ is nondecreasing (resp. nonincreasing). The effect may remain, for instance,

\footnotetext{
${ }^{21}$ As underlined above this case is consistent with the interpretation of $\eta$ as a cautiousness parameter.

${ }^{22}$ In his setting, a value of $\beta$ above 1 corresponds to an exogeneous negative discount of future consumption and does not correspond to a natural behavior. In our setting, the possibly negative discount rate results endogeneously from beliefs heterogeneity.
} 
downwards, if the aggregate probability is pessimistic, and if $B$ is nonincreasing as long as $\left|\mu^{M}\right|$ is "small" which is associated in classical examples to a small dispersion of beliefs.

\subsection{Asset price}

Adopting the same approach as in the standard setting, we easily obtain that for a risky asset with price process $S$ and dividend yield process $e^{*}$,

$$
q_{t}^{*} S_{t}=E_{t}\left[\int_{t}^{T} q_{s}^{*} e_{s}^{*} d s\right]
$$

with $q_{t}^{*}=\bar{M}_{t} B_{t} u^{\prime}\left(t, e_{t}^{*}\right)$, so that the asset price in a heterogeneous beliefs setting is given by

$$
S_{t}[\text { heterogeneous }]=\frac{1}{u^{\prime}\left(t, e_{t}^{*}\right)} E_{t}^{Q}\left[\int_{t}^{T} \frac{B_{s}}{B_{t}} u^{\prime}\left(s, e_{s}^{*}\right) e_{s}^{*} d s\right]
$$

which is to be compared to the following asset price formula in the standard setting

$$
S_{t}[\text { standard }]=\frac{1}{u^{\prime}\left(t, e_{t}^{*}\right)} E_{t}^{P}\left[\int_{t}^{T} u^{\prime}\left(s, e_{s}^{*}\right) e_{s}^{*} d s\right]
$$

As in the risk-free rate analysis, both effects of the change of probability and the discount factor are to be noticed.

If $B$ is nonincreasing which is the case for linear risk tolerance utility functions when $\eta \leq 1$, the effect of the aggregation bias is towards a lowering of the asset price, and in that case,

$$
S_{t}[\text { heterogeneous }] \leq \frac{1}{u^{\prime}\left(t, e_{t}^{*}\right)} E_{t}^{Q}\left[\int_{t}^{T} u^{\prime}\left(s, e_{s}^{*}\right) e_{s}^{*} d s\right]
$$

which means that the asset price under heterogeneous beliefs is lower than the asset price in a model where all investors share the same probability $Q$. The converse effect occurs for general power utility functions with $\eta \geq 1$. The interpretation here again is clear since $B$ represents a discount factor. 
On the other hand, the change of probability effect can be measured through the comparison between $E_{t}^{Q}\left[\int_{t}^{T} u^{\prime}\left(s, e_{s}^{*}\right) e_{s}^{*} d s\right]$ and $E_{t}^{P}\left[\int_{t}^{T} u^{\prime}\left(s, e_{s}^{*}\right) e_{s}^{*} d s\right]$. The effect is towards a raising of the asset price when $E_{t}^{Q}\left[u^{\prime}\left(s, e_{s}^{*}\right) e_{s}^{*}\right] \geq E_{t}^{P}\left[u^{\prime}\left(s, e_{s}^{*}\right) e_{s}^{*}\right]$, which again is related to the pessimism/optimism of the consensus belief $Q$. Indeed, consider the case of power utility functions, $u^{\prime}(t, x)=(\eta x)^{-1 / \eta}$, and suppose that for all $t, \alpha_{t}$ and $\beta_{t}$ are deterministic. Then $E_{t}^{Q}\left[u^{\prime}\left(s, e_{s}^{*}\right) e_{s}^{*}\right]=E_{t}^{Q}\left[\left(e_{s}^{*}\right)^{1-1 / \eta}\right]$, and it is easy to see (See the Appendix, Proof of Inequality (3.6)) that if the consensus probability is pessimistic, i.e. if $\delta^{M} \leq 0$ and if $\eta \leq 1$, then

$$
E_{t}^{Q}\left[\left(e_{s}^{*}\right)^{1-1 / \eta}\right] \geq E_{t}^{P}\left[\left(e_{s}^{*}\right)^{1-1 / \eta}\right]
$$

The same result holds for an optimistic consensus probability and $\eta \geq 1$. For $\delta^{M} \leq 0$ and $\eta \geq 1$ (resp. for $\delta^{M} \geq 0$ and $\eta \leq 1$ ), we obtain $E_{t}^{Q}\left[\left(e_{s}^{*}\right)^{1-1 / \eta}\right] \leq E_{t}^{P}\left[\left(e_{s}^{*}\right)^{1-1 / \eta}\right]$.

\section{Applications}

We shall focus in this section on the case of linear risk tolerance utility functions, and in order to disentangle wealth effect and pure risk aversion effect, we will analyze separately the case of exponential utility functions and the case of power utility functions.

\subsection{Exponential utility functions and the risk aversion effect}

In the case of exponential utility functions, the parameters involved in the expressions of $M, \mu^{M}$ and $\delta^{M}$ do not depend upon the individual characteristics (prices and individual allocations). 
Indeed, we have ${ }^{23}$, for utility functions of the form $u_{i}^{\prime}(t, x)=\exp \left(-\frac{x}{\theta_{i}}\right)$,

$$
\begin{aligned}
M & =\prod_{i=1}^{N}\left(M^{i}\right)^{\theta_{i} / \bar{\theta}}, \quad \delta^{M}=\sum_{i=1}^{N} \frac{\theta_{i}}{\bar{\theta}} \delta^{i}, \\
\mu^{M} & =-\frac{1}{2}\left[\sum_{i=1}^{N} \frac{\theta_{i}}{\bar{\theta}}\left(\delta^{i}\right)^{2}-\left(\sum_{i=1}^{N} \frac{\theta_{i}}{\bar{\theta}} \delta^{i}\right)^{2}\right], \\
\Delta(M P R) & =-\delta^{M}, \quad \Delta(\text { Risk Premium })=-\delta^{M} \sigma^{R}, \quad \Delta\left(r^{f}\right)=-\mu^{M}+\frac{\beta e^{*} \delta^{M}}{\bar{\theta}}
\end{aligned}
$$

The aggregate characteristic $M$ (resp. the aggregate diffusion coefficient $\delta^{M}$ ) overweights the individual beliefs $M^{i}$ (resp. the diffusion coefficients $\delta^{i}$ ) for which $\theta_{i}$ is greater than the average and underweights the beliefs $M^{i}$ (resp. the individual diffusion coefficients $\delta^{i}$ ) for which $\theta_{i}$ is smaller than the average.

If all $\theta_{i}$ are equal, then $M=\prod_{i=1}^{N}\left(M^{i}\right)^{1 / N}$ and $\delta^{M}=\frac{1}{N} \sum_{i=1}^{N} \delta^{i}$ and the impact on the risk premium is simply given by the pessimism/optimism of the "equally-weighted average" investor. If investors are on average optimistic (resp. pessimistic), then the risk premium is lower (resp. higher) than in the standard setting ${ }^{24}$. Furthermore, the impact of beliefs heterogeneity on the interest rate is towards a raising of the interest rate.

Consider now the setting with different risk tolerance parameters $\theta_{i}$. In that case, $\delta^{M}=$ $E_{a}\left[\delta^{i}\right]+\operatorname{cov}_{a}\left[\frac{\theta_{i}}{\theta}, \delta^{i}\right]$ where $E_{a}$ and $\operatorname{cov}_{a}$ denote the agents equally-weighted expectation and covariance. The first effect on the diffusion coefficient $\delta^{M}$ (hence on the risk premium) is given as in the previous case by the average level of optimism/pessimism $E_{a}\left[\delta^{i}\right]$. The second effect is given by the covariance between risk tolerance/aversion and optimism/pessimism. If, for instance, we assume that the more risk tolerant investors are pessimistic and the less risk tolerant investors are optimistic, then we get a higher market price of risk and a lower interest rate (as soon as the dispersion remains moderate). It remains to analyze the validity of such a

\footnotetext{
${ }^{23}$ For a given equilibrium characteristic $x$ (risk premium, market price of risk, risk free rate, etc.) $\Delta(x)$ stands for the difference between the value of $x$ in the heterogeneous beliefs setting and in the standard setting.

${ }^{24}$ In particular, if we suppose that there is no systematic bias in the beliefs, i.e. that $E_{a}\left[\delta^{i}\right] \equiv \frac{1}{N} \sum_{i=1}^{N} \delta^{i}=0$, then we get that the market price of risk remains unchanged with respect to the homogeneous setting.
} 
negative/positive correlation. This could be done through behavioral or psychological empirical studies, and to our knowledge, this question is still open. This could also be done through the introduction in our model of a specific learning process which would lead to such a correlation, and this is left for future research ${ }^{25}$.

\subsection{Power utility functions and initial wealth effect}

We have seen that in the case of power utility functions of the form $u_{i}^{\prime}(t, x)=x^{-\frac{1}{\eta}}$, we have

$$
\begin{aligned}
M^{\eta} & =\sum_{i=1}^{N} \frac{\left(\lambda_{i}\right)^{-\eta}}{\sum_{i=1}^{N}\left(\lambda_{i}\right)^{-\eta}}\left(M^{i}\right)^{\eta}, \quad \delta^{M}=\sum_{i=1}^{N} \frac{y^{*^{i}}}{e^{*}} \delta^{i} \\
\mu^{M} & =\frac{1}{2}(\eta-1)\left[\sum_{i=1}^{N} \frac{y^{*^{i}}}{e^{*}}\left(\delta^{i}\right)^{2}-\left(\sum_{i=1}^{N} \frac{y^{*^{i}}}{e^{*}} \delta^{i}\right)^{2}\right], \\
\Delta(M P R) & \left.=-\delta^{M}, \quad \Delta \text { (Risk Premium }\right)=-\delta^{M} \sigma^{R}, \quad \Delta\left(r^{f}\right)=-\mu^{M}+\frac{\beta}{\eta} \delta^{M} .
\end{aligned}
$$

Let us analyze these formulas in the specific framework of two agents, with constant diffusion coefficients $\delta^{i}$. The weights in these formulas are still proportional to the individual risk tolerances or to $\left(\lambda_{i}\right)^{-\eta}\left(M^{i}\right)^{\eta}$. However these risk tolerances depend on the individual equilibrium allocations and are time and state dependent. In the next, we shall try to provide a simple way to estimate the impact on the market price of risk.

In the case of logarithmic utility functions $(\eta=1)$, we easily obtain that $1 / \lambda_{i}=w_{i}$, where $w_{i}=E\left[\int_{0}^{T} q_{t} e_{t}^{*^{i}} d t\right]$ denotes investor $i^{\prime} \mathrm{s}$ initial wealth and the consensus belief is a wealthweighted average of the individual beliefs (see Rubinstein, 1976). We prove in the next that this last result remains true, in an approximate way, for power utility functions.

We start by proving that $\left(\frac{\lambda_{1}}{\lambda_{2}}\right)^{-\eta}$ is approximately equal to $\frac{w_{1}}{w_{2}}$. More precisely,

Lemma 4.1. When the parameters $\left(\delta_{1}, \delta_{2}, \alpha, \beta\right)$ are constant, we have

$$
\frac{w_{1}}{w_{2}} \exp k_{2} \varepsilon \eta \leq\left(\frac{\lambda_{1}}{\lambda_{2}}\right)^{-\eta} \leq \frac{w_{1}}{w_{2}} \exp k_{1} \varepsilon \eta
$$

\footnotetext{
${ }^{25}$ Once again, notice that the introduction of such a learning process is consistent with our framework.
} 
where $\varepsilon$ is given by $\frac{\left|\delta_{1}-\delta_{2}\right|}{2}$ and where $k_{1}$ and $k_{2}$ are positive constants.

This means that for a small dispersion of beliefs (i.e. for small $\varepsilon$ ), the aggregate characteristic $M$ can be approximated by the wealth-weighted average of the individual beliefs, given by $\left[\left(\frac{w_{1}}{w_{1}+w_{2}}\right)\left(M^{1}\right)^{\eta}+\left(\frac{w_{2}}{w_{1}+w_{2}}\right)\left(M^{2}\right)^{\eta}\right]^{1 / \eta}$. The consensus characteristic reflects then the optimism/pessimism of the wealthier agent. Note that in the case of two agents with the same initial wealth, we prove (see the Appendix) that there is a bias towards optimism (resp. pessimism) when $\eta<1$ (resp. $\eta>1$ ) even if this effect is negligible since as stated in Lemma 4.1 the relative weight $\left(\frac{\lambda_{1}}{\lambda_{2}}\right)^{-\eta}$ is near 1 .

As a direct consequence of Lemma 4.1, we obtain numerically that for a large range of possible values for $\eta$ (namely $\eta \in] 0.1 ; 1.1\left[\right.$ ), for $\varepsilon(\varepsilon \in] 0 ; 0.33[)$ and for $\frac{w_{1}}{w_{2}}\left(\frac{w_{1}}{w_{2}} \in\right] 1 ; 9[$ ), the aggregate characteristic is approximated by the wealth-weighted average of the individual beliefs with a reasonable precision. The relative error made is mostly around $1 \%$ and always lower than $5 \%$.

As far as the risk premium is concerned, we have seen, in Section 3, that the impact of the heterogeneity of beliefs is measured by $\sigma^{R} \delta^{M}$, where $\sigma^{R}$ denotes the volatility of the asset under consideration. We prove that the expected value of the consensus belief diffusion coefficient $\delta^{M}$ is approximately given by the wealth-weighted average of the individual belief diffusion coefficients $\delta^{i}$. The following Lemma gives an upper bound for the error made by this approximation in the specific case of no systematic bias, i.e. when $E_{a}\left[\delta^{i}\right]=0, \delta_{1}=-\delta_{2}=\varepsilon$. A similar approximation can be obtained in the general case.

Lemma 4.2. If $\delta_{1}=-\delta_{2}=\varepsilon \in \mathbb{R}_{+}^{*}$, then for some positive constants $k_{3}, k_{4}, k_{5}$, and $k_{6}$ we have $\eta^{2} \varepsilon^{3} k_{5}+k_{6} \varepsilon\left(\exp k_{2} \varepsilon \eta-1\right) \leq E\left[\delta^{M}\right]-\left(\frac{w_{1}}{w_{1}+w_{2}} \delta_{1}+\frac{w_{2}}{w_{1}+w_{2}} \delta_{2}\right) \leq \eta^{2} \varepsilon^{3} k_{3}+k_{4} \varepsilon\left(\exp k_{1} \varepsilon \eta-1\right)$

For the same range of possible values for $\eta, \varepsilon$ and $\frac{w_{1}}{w_{2}}$ as above the maximum approximation error is around 0.01. The wealth-weighted average of the individual beliefs diffusion coefficients is then a good approximation of the expected value of the aggregate belief diffusion coefficient. This permits a simple analysis of the impact of the introduction of beliefs heterogeneity on the 
risk premium.

The consensus belief diffusion coefficient reflects the optimism/pessimism of the "wealthier" agent. If wealth is positively correlated with optimism we obtain a lower expected risk premium for assets with high beliefs dispersion ${ }^{26}$. Prices will reflect the optimistic view, even though short sale constraints are not part of our model. This result is consistent with the empirical study of Diether et al. (2002), who "provide evidence that stocks with higher dispersion in analysts earnings forecasts earn lower future returns than otherwise similar stocks".

For example, let us suppose that the volatility $\beta$ of aggregate wealth $e^{*}$ is equal to $5 \%$, and that its drift $\alpha$ is equal to $3 \%$, and let us assume that the optimists (resp. pessimists) belief for the drift $\alpha_{1}$ (resp. $\alpha_{2}$ ) is equal to $5 \%$ (resp. $\left.1 \%\right)^{27}$. Let us also suppose that the optimists wealth is three times larger than the pessimists one. The risk premium is then $3 \%$ lower than in the standard setting for an asset whose volatility is equal to $15 \%{ }^{28}$.

Notice that a negative correlation between optimism and wealth would lead to a more pessimistic consensus belief and would raise the risk premium.

If we now introduce some bias in the investors beliefs, and suppose as in e.g. Abel (2000) that investors are on average pessimistic (i.e. that $\bar{\delta}=E_{a}\left[\delta^{i}\right]<0$ ), then we obtain that the aggregate diffusion coefficient is in expected value approximated by $\bar{\delta}+\left(\frac{w_{1}-w_{2}}{w_{1}+w_{2}}\right) \varepsilon$, which remains negative if the dispersion of beliefs is small. As above, the risk premium is lower for stocks with higher beliefs dispersion. But in this situation, the risk premium is higher and the

\footnotetext{
${ }^{26}$ For the sake of simplicity we only considered one source of risk. However, all results can be easily generalized to multidimensional sources of risk. For instance, if we assume that the total wealth follows

$$
d e_{t}^{*}=\alpha_{t} e_{t}^{*} d t+\beta_{t}^{1} e_{t}^{*} d W_{t}^{1}+\beta_{t}^{2} e_{t}^{*} d W_{t}^{2}, \quad \beta>0
$$
}

then agents may disagree on each source of risk and will have beliefs of the following form

$$
d M_{t}^{i}=\delta_{t}^{i, 1} M_{t}^{i} d W_{t}^{1}+\delta_{t}^{i, 2} M_{t}^{i} d W_{t}^{2}, \quad M_{0}^{i}=1 .
$$

The consensus belief will be given by

$$
d M_{t}=\sum \tau_{i} \delta_{t}^{i, 1} M_{t} d W_{t}^{1}+\sum \tau_{i} \delta_{t}^{i, 2} M_{t} d W_{t}^{2}, \quad M_{0}=1
$$

and the impact on the risk premium for an asset that is only affected by the first (resp. second) source of risk is given by $\sum \tau_{i} \delta_{t}^{i, 1}$ (resp. $\sum \tau_{i} \delta_{t}^{i, 2}$ ).

${ }^{27}$ This corresponds to a nonsystematic bias situation $(\bar{\delta}=0)$ with $\Delta=\frac{\alpha_{1}-\alpha_{2}}{2 \beta}=0.4$.

${ }^{28}$ Indeed, it is immediate that the impact $-\sigma^{R} \delta^{M}$ on the risk premium is equal to $-\frac{\sigma^{R}}{\beta}\left(\frac{\alpha_{1} w_{1}+\alpha_{2} w_{2}}{w_{1}+w_{2}}-\alpha\right)$. 
risk-free rate lower than in the standard setting, which is interesting in light of the risk premium and risk-free rate puzzles.

In order to "calibrate" the model with market data, let us assume that $\beta=3.6 \%, \alpha=1.8 \%$ and $\sigma^{R}=16.8 \%$. These numbers correspond to the observed volatility and drift for the US consumption during the last century and to the market portfolio volatility during the same period. In Table 1, we provide for these specifications and for different values of the wealth distribution parameter $\frac{w_{1}}{w_{1}+w_{2}}$, of the cautiousness parameter $\eta$, of the average belief $\bar{\delta}$ and of belief dispersion $\varepsilon$, the equilibrium risk premium and risk-free rate. If $\bar{\delta}=\varepsilon=0$, there is no belief heterogeneity and the common belief corresponds to the true probability (rational expectations). In that case, the estimated risk premium is too low. When $\eta$ decreases, it is possible to obtain higher values for the risk premium; however the risk-free rate becomes too high. These observations correspond to the classical risk premium and risk-free rate puzzles (see Kocherlakota, 1996, for a survey on these "puzzles").

If we introduce some pessimism $(\bar{\delta}<0)$ without belief dispersion, we obtain higher risk premia and lower risk-free rates: the introduction of some pessimism might be a possible partial explanation of the puzzles (see e.g. Abel (2002)).

Finally, if we introduce some pessimism $(\bar{\delta}<0)$ as well as some belief dispersion, we can obtain risk premium and risk-free rate levels that are compatible with historical data for both cases, $\eta<1$ and $\eta \geq 1$. Furthermore, for $\eta<1$, higher belief dispersion leads to a lower risk premium as in Diether et al.(2002).

\section{Concluding and additional remarks}

In this paper, we provide an aggregation procedure which allows us to analyze a market equilibrium model with heterogeneous beliefs, to rewrite in a simple way the equilibrium characteristics (market price of risk, risk premium, risk-free rate) in a heterogeneous beliefs framework and to compare them with an otherwise similar standard setting. The impact of belief heterogeneity on market price of risk is characterized by the risk tolerance weighted average belief. For power 
utility functions, this average can be approximated by the wealth weighted average belief. Furthermore, there is a way to parameterize the heterogeneous beliefs model in order to obtain globally higher risk premia and lower risk-free rates.

Our aggregation procedure enables us to retrieve in a simple and intuitive way well known results of the financial literature on portfolio constraints. For instance, let us consider a model where investors share common beliefs but are submitted to possible short-sale constraints. Intuitively, the equilibrium prices and allocations in such a model are the same as in a model without short-sale constraint but with heterogeneous beliefs. It suffices to replace the beliefs of the investors for which the short-sale constraint (in the initial model) is binding by well chosen more optimistic beliefs and to leave others beliefs unchanged. The consensus consumer is then optimistic and the impact on the MPR and risk-free rate follows: the short sales constraints lead to a lower market price of risk and to a higher risk-free rate. The case of borrowing constraints can be analyzed similarly and leads to opposite results. In Basak and Cuoco (1998), the authors consider a two-agent equilibrium model with restricted market participation. The first agent does not have access to the risky asset market and the second one is not restricted. Without market restrictions, the first agent would buy shares of the risky asset. It is then easy to see that the equilibrium prices and allocations in such a model are the same as in a model without market restrictions, where the first agent's belief is replaced by a well-chosen, more pessimistic one. The aggregate probability is then pessimistic, which leads to a higher risk premium and a lower risk-free rate. 


\section{Appendix}

Proof of Proposition 1. Since $q^{*}$ is an interior equilibrium price process relative to the beliefs $\left(M^{i}\right)$, and the income processes $e^{*^{i}}$, we know that $\sum_{i=1}^{N} y^{*^{i}}=e^{*}$ and that there exist positive Lagrange multipliers $\left(\lambda_{i}\right)$ such that for all $i$ and for all $t$,

$$
M_{t}^{i} u_{i}^{\prime}\left(t, y_{t}^{*^{i}}\right)=\lambda_{i} q_{t}^{*}
$$

We consider the maximization problem

$$
\left(\mathcal{P}^{\lambda}(t, \omega)\right): \max _{\sum_{i=1}^{N} x_{i} \leq e_{t}^{*}(\omega)} \sum \frac{1}{\lambda_{i}} u_{i}\left(t, x_{i}\right) .
$$

Since all utility functions are increasing and strictly concave and take values in $\mathbb{R} \cup\{-\infty\}$, for each $t$ and each $\omega$ this program admits a unique solution $\left(y^{i, \lambda}(t, \omega)\right)$ and we have $\sum_{i=1}^{N} y^{i, \lambda}(t, \omega)=$ $e^{*}(t, \omega)$. Furthermore, the process $\left(\frac{1}{\lambda_{i}} u_{i}^{\prime}\left(t, y_{t}^{i, \lambda}\right)\right)_{t}$ is independent from $i$. We denote this process by $p^{(\lambda)}$. Letting $M^{(\lambda)} \equiv \frac{q^{*}}{p^{(\lambda)}}$, we then have for all $i$ and for all $t$

$$
M_{t}^{(\lambda)} u_{i}^{\prime}\left(t, y_{t}^{i,(\lambda)}\right)=M_{t}^{i} u_{i}^{\prime}\left(t, y_{t}^{*^{i}}\right)
$$

Let us take $\bar{M}=M^{(\lambda)}, \bar{y}^{i}=y^{i,(\lambda)}$, and $\bar{e}^{i}=e^{*^{i}}-y^{*^{i}}+\bar{y}^{i}$. The process $\bar{M}$ is adapted and positive. Moreover, at date $t=0$, we have for all $i, M_{0}^{i}=1$, and $\sum_{i=1}^{N} \bar{y}_{0}^{i}=\sum_{i=1}^{N} y_{0}^{*^{i}}=e_{0}^{*}$, so that $\overline{M_{0}}=1$.

As far as uniqueness is concerned, notice that any process $y^{i}$ such that $\sum_{i=1}^{N} y^{i}=e^{*}$ and

$$
M_{t} u_{i}^{\prime}\left(t, y_{t}^{i}\right)=M_{t}^{i} u_{i}^{\prime}\left(t, y_{t}^{*^{i}}\right)
$$

for some positive process $M$ is a solution of the maximization problem $\left(\mathcal{P}^{\lambda}\right)$, which, as seen above, admits a unique solution.

Proof of Proposition 2. Similar to the proof of the analogous result in a standard setting. 
Proof of Example 2.1 Since the representative utility function $u_{\lambda}$ is given by

$$
u_{\lambda}(t, x)=\max _{\sum_{i=1}^{N} x_{i} \leq x} \sum_{i=1}^{N} \frac{1}{\lambda_{i}} u_{i}\left(t, x_{i}\right)
$$

the expression of $u_{\lambda}$ in the specific setting of linear risk tolerance utility functions is obtained, as in the standard case (see e.g. Huang-Litzenberger, 1988), by using $\frac{1}{\lambda_{i}} u_{i}^{\prime}\left(t, x_{i}\right)=u^{\prime}(t, x)$ with $\sum_{i=1}^{N} x_{i}=x$.

The expression of $M$ is obtained by using $M_{t} u_{i}^{\prime}\left(t, \bar{y}_{t}^{i}\right)=M_{t}^{i} u_{i}^{\prime}\left(t, y_{t}^{*^{i}}\right)$, as well as

$$
\sum_{i=1}^{N} y^{*^{i}}=\sum_{i=1}^{N} \bar{y}^{i}=e^{*}
$$

Indeed, in the case of exponential utility functions, we have for all $i$,

$$
M^{i} \exp \left(-\frac{y^{*^{i}}}{\theta_{i}}\right)=M \exp \left(-\frac{\bar{y}^{i}}{\theta_{i}}\right)
$$

hence

$$
\prod_{i=1}^{N}\left(M^{i}\right)^{\theta_{i}} \exp \left(-\sum_{i=1}^{N} y^{*^{i}}\right)=M^{\bar{\theta}} \exp \left(-\sum_{i=1}^{N} \bar{y}^{i}\right)
$$

or equivalently

$$
M=\prod_{i=1}^{N}\left(M^{i}\right)^{\frac{\theta_{i}}{\bar{\theta}}}
$$

In the case of power utility functions, we get for all $i$,

$$
M^{i}\left(\theta_{i}+\eta y^{*^{i}}\right)^{-1 / \eta}=M\left(\theta_{i}+\eta \bar{y}^{i}\right)^{-1 / \eta}=\frac{\lambda_{i}}{b_{i}} M\left(\bar{\theta}+\eta e^{*}\right)^{-1 / \eta} b
$$

hence

$$
\left(M^{i}\right)^{\eta}\left(\frac{b_{i}}{\lambda_{i}}\right)^{\eta}=M^{\eta}\left(\bar{\theta}+\eta e^{*}\right)^{-1} b^{\eta}\left(\theta_{i}+\eta y^{*^{i}}\right)
$$


and

$$
\begin{aligned}
M & =\left[\sum_{i=1}^{N} \frac{\left(\frac{b_{i}}{\lambda_{i}}\right)^{\eta}}{\sum_{j=1}^{N}\left(\frac{b_{j}}{\lambda_{j}}\right)^{\eta}}\left(M^{i}\right)^{\eta}\right]^{1 / \eta} \\
& =\left[\sum_{i=1}^{N} \tau_{i}\left(M^{i}\right)^{-\eta}\right]^{-1 / \eta} .
\end{aligned}
$$

Proof of Proposition 3. We know by Proposition 2 that there exists a consensus consumer with consensus characteristic $M$ and utility function $u$ such that $M_{t} u^{\prime}\left(t, e_{t}^{*}\right)=q_{t}^{*}$. Since $d M_{t}=$ $M_{t}\left[\mu^{M}(t) d t+\delta^{M}(t) d W_{t}\right]$ with $M_{0}=1$, we have

$$
M_{t}=\exp \left[\int_{0}^{t} \delta_{u}^{M} d W_{u}+\int_{0}^{t}\left(\mu_{u}^{M}-\frac{1}{2}\left(\delta_{u}^{M}\right)^{2}\right) d u\right]
$$

hence with the notations of the Proposition, $M_{t}=B_{t} \bar{M}_{t}$.

Proof of Proposition 4. When $-\frac{u_{i}^{\prime}(t, x)}{u_{i}^{\prime \prime}(t, x)}=\theta_{i}>0$, then we know from Example (2.1) that $M_{t}=\prod_{i=1}^{N}\left(M_{t}^{i}\right)^{\theta_{i} / \bar{\theta}}$. Since $M_{t}^{i}=\exp \left[\int_{0}^{t} \delta_{u}^{i} d W_{u}-\frac{1}{2} \int_{0}^{t}\left(\delta_{u}^{i}\right)^{2} d u\right]$, we have

$$
\prod_{i=1}^{N}\left(M_{t}^{i}\right)^{\theta_{i} / \bar{\theta}}=\exp \left[\int_{0}^{t} \sum_{i=1}^{N} \frac{\theta_{i}}{\bar{\theta}} \delta_{u}^{i} d W_{u}-\frac{1}{2} \int_{0}^{t} \sum_{i=1}^{N} \frac{\theta_{i}}{\bar{\theta}}\left(\delta_{u}^{i}\right)^{2} d u\right]
$$

hence by Itô's Lemma,

$$
\left\{\begin{array}{c}
\delta^{M}=\sum_{i=1}^{N} \frac{\theta_{i}}{\theta} \delta^{i} \\
\mu^{M}=-\frac{1}{2}\left[\sum_{i=1}^{N} \frac{\theta_{i}}{\theta}\left(\delta^{i}\right)^{2}-\left(\sum_{i=1}^{N} \frac{\theta_{i}}{\theta} \delta^{i}\right)^{2}\right]
\end{array}\right.
$$

When $-\frac{u_{i}^{\prime}(t, x)}{u_{i}^{\prime}(t, x)}=\theta_{i}+\eta x$ for $\eta \neq 0$, the result is obtained in the same way, by applying Itô's Lemma to the expression of the consensus belief $M=\left[\sum_{i=1}^{N} \tau_{i}\left(M^{i}\right)^{-\eta}\right]^{-\frac{1}{\eta}}$ obtained in Example 2.1 . 
Proof of Proposition 5. Since $q^{*}$ is a state price density, the price process $S$ must be such that $q^{*} S$ is a $P$-martingale so that as in the classical case (see, e.g. Duffie, 1996, or HuangLitzenberger, 1988) we have $\mu^{R}-r^{f}=-\sigma_{q^{*}} \sigma^{R}$ and $r^{f}=-\mu_{q^{*}}$. Now, since $q_{t}^{*}=M_{t} u^{\prime}\left(t, e_{t}^{*}\right)$ we easily get, through Itô's Lemma,

$$
M P R_{t}[\text { heterogeneous }]=-\frac{u^{\prime \prime}\left(t, e_{t}^{*}\right)}{u^{\prime}\left(t, e_{t}^{*}\right)} \beta_{t} e_{t}^{*}-\delta_{t}^{M}
$$

and

$$
r_{t}^{f}[\text { heterogeneous }]=r_{t}^{f}[\text { standard }]-\mu_{t}^{M}+\delta_{t}^{M}\left(-\frac{u^{\prime \prime}\left(t, e_{t}^{*}\right)}{u^{\prime}\left(t, e_{t}^{*}\right)} \beta_{t} e_{t}^{*}\right) .
$$

Proof of Inequality 3.6 Since $d e_{t}^{*}=e_{t}^{*}\left(\alpha_{t} d t+\beta_{t} d W_{t}\right)$, we have

$$
e_{t}^{*}=e_{0}^{*} \exp \left[\int_{0}^{t}\left(\alpha_{u}-\frac{1}{2} \beta_{u}^{2}\right) d u+\int_{0}^{t} \beta_{u} d W_{u}\right]
$$

Letting $W_{t}^{Q} \equiv W_{t}-\int_{0}^{t} \delta^{M}(u) d u$, we know, by Girsanov's Lemma, that $W^{Q}$ is a $Q$-Brownian motion, and

$$
\left(\frac{e_{s}^{*}}{e_{t}^{*}}\right)^{1-1 / \eta}=\exp (1-1 / \eta)\left[\int_{t}^{s}\left(\alpha_{u}-\frac{1}{2} \beta_{u}^{2}\right) d u+\int_{t}^{s} \beta_{u} d W_{u}^{Q}\right] \exp (1-1 / \eta) \int_{t}^{s} \beta_{u} \delta^{M}(u) d u
$$

If $\delta^{M} \leq 0$, and $\eta \leq 1$, then $\exp (1-1 / \eta) \int_{t}^{s} \beta_{u} \delta^{M}(u) d u \geq 1$, hence,

$$
\begin{aligned}
E_{t}^{Q}\left[\left(e_{s}^{*}\right)^{1-1 / \eta}\right] & =\left(e_{t}^{*}\right)^{1-1 / \eta} E_{t}^{Q}\left[\left(\frac{e_{s}^{*}}{e_{t}^{*}}\right)^{1-1 / \eta}\right] \\
& \geq\left(e_{t}^{*}\right)^{1-1 / \eta} E_{t}^{Q}\left[\exp (1-1 / \eta)\left[\int_{t}^{s}\left(\alpha_{u}-\frac{1}{2} \beta_{u}^{2}\right) d u+\int_{t}^{s} \beta_{u} d W_{u}^{Q}\right]\right] \\
& \geq\left(e_{t}^{*}\right)^{1-1 / \eta} E_{t}^{P}\left[\exp (1-1 / \eta)\left[\int_{t}^{s}\left(\alpha_{u}-\frac{1}{2} \beta_{u}^{2}\right) d u+\int_{t}^{s} \beta_{u} d W_{u}\right]\right] \\
& \geq E_{t}^{P}\left[\left(e_{s}^{*}\right)^{1-1 / \eta}\right] .
\end{aligned}
$$

The same approach leads to the same result (resp. the opposite result, $E_{t}^{Q}\left[\left(e_{s}^{*}\right)^{1-1 / \eta}\right] \leq$ 
$\left.E_{t}^{P}\left[\left(e_{s}^{*}\right)^{1-1 / \eta}\right]\right)$ when $\delta^{M} \geq 0$ and $\eta \geq 1\left(\right.$ resp. $\delta^{M} \geq 0$ and $\eta \leq 1, \delta^{M} \leq 0$ and $\left.\eta \geq 1\right)$.

Proof of Lemma 4.1 In equilibrium, we have

$$
\begin{aligned}
\frac{1}{\lambda_{1}} M_{t}^{1} u_{1}^{\prime}\left(t, y_{t}^{*^{1}}\right) & =\frac{1}{\lambda_{2}} M_{t}^{2} u_{2}^{\prime}\left(t, y_{t}^{*^{2}}\right)=q_{t}^{*} \\
y_{t}^{*^{1}}+y_{t}^{*^{2}} & =e^{*} \\
w_{2} E\left[\int_{0}^{T} q_{t} y_{t}^{*^{1}} d t\right] & =w_{1} E\left[\int_{0}^{T} q_{t} y_{t}^{*^{2}} d t\right]
\end{aligned}
$$

Equations (5.3) and (5.4) lead to

$$
\left\{\begin{aligned}
y^{*^{1}} & =e^{*} \frac{\left(M^{1} / \lambda_{1}\right)^{\eta}}{\left(M^{1} / \lambda_{1}\right)^{\eta}+\left(M^{2} / \lambda_{2}\right)^{\eta}} \\
y^{*^{2}} & =e^{*} \frac{\left(M^{2} / \lambda_{2}\right)^{\eta}}{\left(M^{1} / \lambda_{1}\right)^{\eta}+\left(M^{2} / \lambda_{2}\right)^{\eta}}
\end{aligned}\right.
$$

and Equation (5.5) then implies that

$$
E\left[\int_{0}^{T}\left(e_{t}^{*}\right)^{1-1 / \eta} \frac{w_{2}\left(M^{1}(t) / \lambda_{1}\right)^{\eta}-w_{1}\left(M^{2}(t) / \lambda_{2}\right)^{\eta}}{\left\{\left(M^{1}(t) / \lambda_{1}\right)^{\eta}+\left(M^{2}(t) / \lambda_{2}\right)^{\eta}\right\}^{1-1 / \eta}} d t\right]=0
$$

Let $A \equiv \frac{w_{2}\left(M^{1} / \lambda_{1}\right)^{\eta}-w_{1}\left(M^{2} / \lambda_{2}\right)^{\eta}}{\left\{\left(M^{1} / \lambda_{1}\right)^{\eta}+\left(M^{2} / \lambda_{2}\right)^{\eta}\right\}^{1-1 / \eta}}$. Then

$$
A=\left(\frac{M^{1}}{\lambda_{1}}\right) \frac{w_{2}-w_{1} X}{\{1+X\}^{1-1 / \eta}}
$$

for $X=\left(\frac{M^{2}}{M^{1}} \frac{\lambda_{1}}{\lambda_{2}}\right)^{\eta}$. We first consider the case $\left(1-\frac{1}{\eta}\right) \geq 0$. It is easy to verify that

$$
\frac{w_{2}-w_{1} X}{\{1+X\}^{1-1 / \eta}} \geq \frac{w_{2}-w_{1} X}{\left\{1+\frac{w_{2}}{w_{1}}\right\}^{1-1 / \eta}}
$$

Now, Equation (5.6) implies that

$$
E\left[\int_{0}^{T}\left(e_{t}^{*}\right)^{1-1 / \eta}\left(\frac{M_{t}^{1}}{\lambda_{1}}\right)\left(\frac{w_{2}-w_{1} X_{t}}{\left\{1+\frac{w_{2}}{w_{1}}\right\}^{1-1 / \eta}}\right) d t\right] \leq 0
$$


hence

$$
\left(\frac{\lambda_{1}}{\lambda_{2}}\right)^{-\eta} \frac{w_{2}}{w_{1}} \leq \frac{E\left[\int_{0}^{T}\left(e_{t}^{*}\right)^{1-1 / \eta} M_{t}^{1}\left(\left(\frac{M_{t}^{2}}{M_{t}^{1}}\right)^{\eta}\right) d t\right]}{E\left[\int_{0}^{T}\left(e_{t}^{*}\right)^{1-1 / \eta} M_{t}^{1} d t\right]}
$$

We obtain analogously that

$$
A=\left(\frac{M^{2}}{\lambda_{2}}\right) \frac{w_{2} / X-w_{1}}{\{1 / X+1\}^{1-1 / \eta}}
$$

with

$$
\frac{w_{2} / X-w_{1}}{\left\{1 / X_{t}+1\right\}^{1-1 / \eta}} \leq \frac{w_{2} / X-w_{1}}{\left\{1+\frac{w_{1}}{w_{2}}\right\}^{1-1 / \eta}}
$$

hence

$$
\left(\frac{\lambda_{1}}{\lambda_{2}}\right)^{-\eta} \frac{w_{2}}{w_{1}} \geq \frac{E\left[\int_{0}^{T}\left(e_{t}^{*}\right)^{1-1 / \eta} M_{t}^{2} d t\right]}{E\left[\int_{0}^{T}\left(e_{t}^{*}\right)^{1-1 / \eta} M_{t}^{2}\left(\left(\frac{M_{t}^{1}}{M_{t}^{2}}\right)^{\eta}\right) d t\right]} .
$$

Now,

$$
\begin{aligned}
& M_{t}^{1}=\exp (\delta+\varepsilon) W_{t}-\frac{1}{2}(\delta+\varepsilon)^{2} t \\
& M_{t}^{2}=\exp (\delta-\varepsilon) W_{t}-\frac{1}{2}(\delta-\varepsilon)^{2} t
\end{aligned}
$$

and

$$
E\left[\int_{0}^{T}\left(e_{t}^{*}\right)^{1-1 / \eta} M_{t}^{1} d t\right]=\int_{0}^{T} E\left[\left(e_{t}^{*}\right)^{1-1 / \eta} M_{t}^{1}\right] d t=\int_{0}^{T}(\exp a t) d t
$$

with $a=\frac{1}{2}\left[\delta+\varepsilon+\beta\left(1-\frac{1}{\eta}\right)\right]^{2}+\left(\alpha-\frac{1}{2} \beta^{2}\right)\left(1-\frac{1}{\eta}\right)-\frac{1}{2}(\delta+\varepsilon)^{2}$. Besides,

$$
E\left[\int_{0}^{T}\left(e_{t}^{*}\right)^{1-1 / \eta} M_{t}^{1}\left(\left(\frac{M_{t}^{2}}{M_{t}^{1}}\right)^{\eta}\right) d t\right]=\int_{0}^{T}(\exp b t) d t
$$

with $b=a+2\left(1-\frac{1}{\eta}\right) \varepsilon \eta(\varepsilon \eta-\beta)$. If $b-a \leq 0$, then

$$
\frac{E\left[\int_{0}^{T}\left(e_{t}^{*}\right)^{1-1 / \eta} M_{t}^{1}\left(\left(\frac{M_{t}^{2}}{M_{t}^{1}}\right)^{\eta}\right) d t\right]}{E\left[\int_{0}^{T}\left(e_{t}^{*}\right)^{1-1 / \eta} M_{t}^{1} d t\right]} \leq 1
$$


and if $b-a \geq 0$, then

$$
\frac{E\left[\int_{0}^{T}\left(e_{t}^{*}\right)^{1-1 / \eta} M_{t}^{1}\left(\left(\frac{M_{t}^{2}}{M_{t}^{1}}\right)^{\eta}\right) d t\right]}{E\left[\int_{0}^{T}\left(e_{t}^{*}\right)^{1-1 / \eta} M_{t}^{1} d t\right]} \leq \exp (b-a) T \leq \exp 2\left(1-\frac{1}{\eta}\right) \varepsilon \eta(\varepsilon \eta-\beta) T
$$

hence $\left(\frac{\lambda_{1}}{\lambda_{2}}\right)^{-\eta} \leq \frac{w_{1}}{w_{2}} \max \left(1, \exp \left\{2\left(1-\frac{1}{\eta}\right) \varepsilon \eta(\varepsilon \eta-\beta)\right\}\right)$. The other inequality is obtained similarly, and the same approach leads to the result for $\left(1-\frac{1}{\eta}\right) \leq 0$.

Proof of the following result: If $\delta_{1}=-\delta_{2}=\varepsilon \in \mathbb{R}_{+}^{*}$, if $\left(\alpha_{t}, \beta_{t}\right) \in \mathbb{R}^{2}$, and if the investors are endowed with the same initial wealth, then

$$
\lambda_{1}<\lambda_{2}, \text { for } \eta<1, \quad \lambda_{1}>\lambda_{2}, \text { for } \eta>1, \quad \lambda_{1}=\lambda_{2}, \text { for } \eta=1
$$

Proof. Proceeding as in the proof of Lemma 4.1, we obtain Equation (5.6) with $w_{1}=w_{2}$, namely

$$
A \equiv E\left[\int_{0}^{T}\left(e_{t}^{*}\right)^{1-1 / \eta} \frac{\left(M^{1} / \lambda_{1}\right)^{\eta}-\left(M^{2} / \lambda_{2}\right)^{\eta}}{\left\{\left(M^{1} / \lambda_{1}\right)^{\eta}+\left(M^{2} / \lambda_{2}\right)^{\eta}\right\}^{1-1 / \eta}} d t\right]=0 .
$$

It is immediate that $A$ can be written in the form $A=\frac{1}{\lambda_{1}} x^{-1 / \eta} g(x)$ with $x^{2}=\left(\frac{\lambda_{2}}{\lambda_{1}}\right)^{\eta}$ and

$$
g(x)=E\left[\int_{0}^{T}\left(e_{t}^{*}\right)^{1-1 / \eta} \frac{x\left(M_{t}^{1}\right)^{\eta}-\frac{1}{x}\left(M_{t}^{2}\right)^{\eta}}{\left\{x\left(M_{t}^{1}\right)^{\eta}+\frac{1}{x}\left(M_{t}^{2}\right)^{\eta}\right\}^{1-1 / \eta}} d t\right] .
$$

For $\eta<1$, we show that $\lambda_{1} \leq \lambda_{2}$. We prove 1) that $g(1) \leq 0$, and 2) that $g$ is increasing with $x$, which implies that for $\lambda_{1}>\lambda_{2}$ we would have $A<0$, which is impossible. We have

$$
g^{\prime}(x)=E\left[\int_{0}^{T} \frac{x\left(\left(M_{t}^{1}\right)^{\eta}+\left(M_{t}^{2}\right)^{\eta} / x^{2}\right)^{2}-x\left(\left(M_{t}^{1}\right)^{\eta}-\left(M_{t}^{2}\right)^{\eta} / x^{2}\right)^{2}(1-1 / \eta)}{\left(x\left(M_{t}^{1}\right)^{\eta}+\left(M_{t}^{2}\right)^{\eta} / x\right)^{2-1 / \eta}}\left(e_{t}^{*}\right)^{1-1 / \eta} d t\right],
$$

which is positive for $\eta<1$ and proves 2). Now, $g(1)=\int_{0}^{T} E\left[\left(e_{t}^{*}\right)^{1-1 / \eta} \frac{\left(M^{1}\right)^{\eta}-\left(M^{2}\right)^{\eta}}{\left\{\left(M^{1}\right)^{\eta}+\left(M^{2}\right)^{\eta}\right\}^{1-1 / \eta}}\right] d t$. With deterministic coefficients, $\left(M^{1}\right)^{\eta}(t)=R(t)\left(e^{*}(t)\right)^{\frac{\eta \varepsilon}{\beta}}$ and $\left(M^{2}\right)^{\eta}(t)=R(t)\left(e^{*}(t)\right)^{-\frac{\eta \varepsilon}{\beta}}$ and it is easy to see that $\frac{\left(M^{1}\right)^{\eta}-\left(M^{2}\right)^{\eta}}{\left\{\left(M^{1}\right)^{\eta}+\left(M^{2}\right)^{\eta}\right\}^{1-1 / \eta}}$ is increasing in $e^{*}$, hence decreasing in $\left(e^{*}\right)^{1-1 / \eta}$, 
leading to

$$
E\left[\left(e_{t}^{*}\right)^{1-1 / \eta} \frac{\left(M_{t}^{1}\right)^{\eta}-\left(M_{t}^{2}\right)^{\eta}}{\left\{\left(M_{t}^{1}\right)^{\eta}+\left(M_{t}^{2}\right)^{\eta}\right\}^{1-1 / \eta}}\right] \leq E\left[\left(e_{t}^{*}\right)^{1-1 / \eta}\right] E\left[\frac{\left(M_{t}^{1}\right)^{\eta}-\left(M_{t}^{2}\right)^{\eta}}{\left\{\left(M_{t}^{1}\right)^{\eta}+\left(M_{t}^{2}\right)^{\eta}\right\}^{1-1 / \eta}}\right] .
$$

Since $E\left[\frac{\left(M_{t}^{1}\right)^{\eta}-\left(M_{t}^{2}\right)^{\eta}}{\left\{\left(M_{t}^{1}\right)^{\eta}+\left(M_{t}^{2}\right)^{\eta}\right\}^{1-1 / \eta}}\right]=0$, we obtain that $g(1) \leq 0$.

Proof of Lemma 4.2 We have $E\left[\delta^{M}\right]-\left(\frac{w_{1}}{w_{1}+w_{2}} \delta_{1}+\frac{w_{2}}{w_{1}+w_{2}} \delta_{2}\right)=E\left[A_{\left(\lambda_{1}^{-\eta}, \lambda_{2}^{-\eta}\right)}\right]+$ $B_{\left(\lambda_{1}^{-\eta}, \lambda_{2}^{-\eta}\right)}$ for $A_{a} \equiv \sum_{i=1}^{2} \frac{a_{i}\left(M_{t}^{i}\right)^{\eta}}{\sum a_{i}\left(M_{t}^{i}\right)^{\eta}} \delta^{i}-\sum_{i=1}^{2} \frac{a_{i}}{\sum a_{i}} \delta^{i}$ and $B_{\left(\lambda_{1}^{-\eta}, \lambda_{2}^{-\eta}\right)}=\sum_{i=1}^{2} \frac{\lambda_{i}^{-\eta}}{\lambda_{1}^{-\eta}+\lambda_{2}^{-\eta}} \delta^{i}-$ $\sum_{i=1}^{2} \frac{w_{i}}{w_{1}+w_{2}} \delta^{i}$. We easily get that $A_{\left(a_{1}, a_{2}\right)}=\frac{a_{1} a_{2}}{\left(a_{1}+a_{2}\right)}(2 \delta) \frac{\left(M_{t}^{1}\right)^{\eta}-\left(M_{t}^{2}\right)^{\eta}}{a_{1}\left(M_{t}^{1}\right)^{\eta}+a_{2}\left(M_{t}^{2}\right)^{\eta}}$. In this setting, we know that $\left(M_{t}^{1}\right)^{\eta}=\exp \left(\eta \delta W_{t}-\frac{1}{2} \eta \delta^{2} t\right)$ and $\left(M_{t}^{2}\right)^{\eta}=\exp \left(-\eta \delta W_{t}-\frac{1}{2} \eta \delta^{2} t\right)$. Then

$$
\begin{aligned}
E\left[\frac{\left(M_{t}^{1}\right)^{\eta}-\left(M_{t}^{2}\right)^{\eta}}{a_{1}\left(M_{t}^{1}\right)^{\eta}+a_{2}\left(M_{t}^{2}\right)^{\eta}}\right] & =\frac{1}{\sqrt{2 \pi t}} \int_{-\infty}^{+\infty} \frac{\exp (\delta \eta x)-\exp (-\delta \eta x)}{a_{1} \exp (\delta \eta x)+a_{2} \exp (-\delta \eta x)} \exp \left(-\frac{x^{2}}{2 t}\right) d t \\
& =\frac{\left(a_{2}-a_{1}\right)}{a_{1} a_{2} \sqrt{2 \pi t}} \int_{0}^{+\infty} \frac{\exp (2 \delta \eta x)+\exp (-2 \delta \eta x)-2}{\exp (2 \delta \eta x)+\exp (-2 \delta \eta x)+\frac{a_{1}}{a_{2}}+\frac{a_{2}}{a_{1}}} \exp \left(-\frac{x^{2}}{2 t}\right) d t
\end{aligned}
$$

Since $\frac{\exp ^{y}+\exp ^{-y}-2}{\exp ^{y}+\exp ^{-y}+c} \leq \max \left(\frac{1}{2+c}, \frac{1}{12}\right) y^{2}$, we obtain

$$
\begin{aligned}
0 & \leq \frac{E\left[A_{a}\right]}{\left(a_{2}-a_{1}\right)} \leq \frac{a_{1} a_{2}}{\left(a_{1}+a_{2}\right)}(2 \delta) \frac{1}{a_{1} a_{2}} \max \left(\frac{1}{2+\frac{a_{1}}{a_{2}}+\frac{a_{2}}{a_{1}}}, \frac{1}{12}\right)(2 \delta \eta)^{2} \frac{1}{\sqrt{2 \pi t}} \int_{0}^{+\infty} \frac{1}{2} x^{2} \exp \left(-\frac{x^{2}}{2 t}\right) d t \\
& \leq \frac{1}{\left(a_{1}+a_{2}\right)} \eta^{2} \delta^{3} \max \left(\frac{1}{3}, \frac{4}{2+\frac{a_{1}}{a_{2}}+\frac{a_{2}}{a_{1}}}\right)
\end{aligned}
$$

Now, $B_{\left(\lambda_{1}^{-\eta}, \lambda_{2}^{-\eta}\right)}=\frac{2 \delta\left[\left(\frac{\lambda_{1}}{\lambda_{2}}\right)^{-\eta} \frac{w_{2}}{w_{1}}-1\right]}{\left(1+\frac{w_{2}}{w_{1}}\right)\left(1+\left(\frac{\lambda_{1}}{\lambda_{2}}\right)^{-\eta}\right)}$. The rest of the proof comes from Lemma 4.1. 


\section{References}

Abel, A., 1989. Asset prices under heterogeneous beliefs: implications for the equity premium. Mimeo, University of Pennsylvania.

Abel, A., 2002. An exploration of the effects of pessimism and doubt on asset returns, Journal of Economic Dynamics and Control, vol. 26(7-8), 1075-1092.

Anderson, E., Hansen L.-P. and T. Sargent, (2000). Robustness, detection and the price of risk. Mimeo, Stanford University.

Aumann, R., 1976. Agreeing to disagree. The Annals of Statistics, 4, 1236-1239.

Basak, S., 2000. A model of dynamic equilibrium asset pricing with heterogeneous beliefs and extraneous risk. Journal of Economic Dynamics and Control, 24, 63-95.

Basak, S. and D. Cuoco, 1998. An equilibrium model with restricted stock market participation, Review of Financial Studies, 11, 309-341.

Breeden, D., 1979. An Intertemporal Asset Pricing Model with Stochastic Consumption and Investment Opportunities. Journal of Financial Economics, 7, 265-296.

Breeden, D. and R. Litzenberger, 1978. Prices of State Contingent Claims Implicit in Option Prices. Journal of Business, 51, 621-651.

Calvet, L., Grandmont, J.-M., and I. Lemaire, 2002. Aggregation of Heterogeneous Beliefs and Asset Pricing in Complete Financial Markets. Working Paper.

Cecchetti, S., Lam, P. and N. Mark, 2000. Asset pricing with distorded beliefs: are equity returns too good to be true? American Economic Review, 90, 787-805.

Constantinidès, G., 1982. Intertemporal asset pricing with heterogeneous consumers and without demand aggregation. Journal of Business, 55, 253-267.

Cragg, J. and B. Malkiel, 1982. Expectations and the Structure of Share Prices. University of Chicago Press, Chicago.

Detemple, J. and S. Murthy, 1994. Intertemporal Asset Pricing with Heterogeneous Beliefs. Journal of Economic Theory, 62, 294-320.

Diether, K., C. Malloy and A. Scherbina, 2002. Differences of opinion and the cross section of stock returns. Journal of Finance, 57, 2113-2141.

Duffie, D., 1996. Dynamic Asset Pricing Theory. Princeton University Press, Princeton.

Duffie, D. and C.-F. Huang, 1985, Implementing Arrow-Debreu Equilibria by Continuous Trading of Few Long-Lived Securities. Econometrica, 53, (6), 1337-1356.

Duffie, D. and W. Zame, 1989, The Consumption-Based Capital Asset Pricing Model. Econometrica, 57, 1279-1297.

Epstein, L. and T. Wang, 1994. Intertemporal asset pricing under Knightian uncertainty. Econometrica, 62 (2), 283-322.

Gallmeyer, M., 2000. Beliefs and volatility. Working paper, Carnegie Mellon University.

Gallmeyer, M. and B. Hollifield, 2002. An examination of heterogeneous beliefs with a short sale constraint. Working paper, Carnegie Mellon University.

Genotte, G., 1986. Optimal portfolio choice under incomplete information. Journal of Finance, 41, 733-746. 
Gollier, C., and M. Kimball, 1996. Toward a systematic approach to the economic effects of uncertainty: Characterizing utility functions, mimeo, University of Toulouse.

Gonedes, N., 1976. Capital Market Equilibrium for a Class of Heterogeneous Expectations in a Two-Parameters World. Journal of Finance, 31, 1-15.

Harris, M. and A. Raviv, 1993. Differences of Opinion Make a Horse Race. Review of Financial Studies, 6 (3), 473-506.

Hansen, L.-P., Sargent, T. and T. Tallarini, 1999. Robust Permanent Income and Pricing. Review of Economic Studies, 66, 873-907.

Huang, C.-F., and R. Litzenberger, 1988. Foundations of Financial Economics, Prentice Hall, Englewood Cliffs, New-Jersey.

Ingersoll, J., 1987. Theory of Financial Decision Making, Rowman and Littlefield, Totowa, New-Jersey.

Jarrow, R., 1980. Heterogeneous Expectations, Restrictions on Short Sale, and Equilibrium Asset Prices. Journal of Finance, 35, 1105-1113.

Karatzas, I. and S. Shreve, 1988. Brownian motion and stochastic calculus. Springer Verlag.

Kocherlakota, N. R., 1996. The Equity Premium: It's Still a Puzzle. Journal of Economic Literature, 34, 42-71.

Lintner, J., 1969. The Aggregation of Investor's Diverse Judgements and Preferences in Purely Competitive Security Markets. Journal of Financial and Quantitative Analysis, 4, 347-400.

Mayshar, J., 1981. Transactions Costs and the Pricing of Assets. Journal of Finance, 36, 583-597.

Mayshar, J., 1983. On Divergence of Opinion and Imperfections in Capital Markets. American Economic Review, 73, 114-128.

Mehra, R., and E. Prescott, 1985. The Equity Premium: A Puzzle. Journal of Monetary Economics, 15, 145-162.

Merton, R., 1973. An Intertemporal Capital Asset Pricing Model. Econometrica, 41, 867-887.

Miller, E. M., 1977. Risk, Uncertainty, and Divergence of Opinion. Journal of Finance, 32, 1151-1168.

Negishi, T., 1960. Welfare Economics and Existence of an Equilibrium for a Competitive Economy. Metroeconomica, 12, 92-97.

Rubinstein, M., 1974. An aggregation theorem for securities markets. Journal of Financial Economics, 1, 225-244.

Rubinstein, M., 1975. Security Market Efficiency in an Arrow-Debreu Economy. American Economic Review, 65, 812-824.

Rubinstein, M., 1976. The Strong Case for the Generalized Logarithmic Utility Model as the Premier Model of Financial Markets. Journal of Finance, 31, 551-571.

Scheinkman, J. A., and W. Xiong, 2003. Overconfidence and Speculative Bubbles. Journal of Political Economy, 111, 1183-1219.

Sharpe, W.F., 1964. Capital Asset Prices: A Theory of Market Equilibrium UnderConditions of Risk. Journal of Finance, 19, 425-442. 
Varian, H., 1985. Divergence of Opinion in Complete Markets. Journal of Finance, 40, 309-317. Varian, H., 1989. Difference of Opinion in Financial Markets. In Financial Risk: Theory, Evidence, and Implications. Stone C.C. (Ed.), Kluwer, Dordrecht, The Netherlands.

Weil, P., 1989. The Equity Premium Puzzle and the risk-free Rate Puzzle. Journal of Monetary Economics, 24, 401-421.

Welch, I., 2000. Views of financial economists on the equity premium and on professional controversies. Working paper, Yale University.

Williams, J. T., 1977. Capital Asset Prices with Heterogeneous Beliefs. Journal of Financial Economics, 5, 219-239.

Zapatero, F., 1998. Effects on Financial Innovations on Market volatility when Beliefs are Heterogeneous. Journal of Economics, Dynamics and Control, 22, 597-626.

Ziegler, A., 2000. Optimal portfolio choice under heterogeneous beliefs. European Finance Review, 4, 1-19. 
TABLE 1

In this table, for $\beta=3.6 \%, \alpha=1.8 \%$ and $\sigma^{R}=16.8 \%$, we report the values of the risk premium and of the risk-free rate for different values of the cautiousness parameter $\eta$, the average belief $\bar{\delta}$, the wealth distribution $\frac{w_{1}}{w_{1}+w_{2}}$ and the belief dispersion $\varepsilon$.

\begin{tabular}{|c|c|c|c|c|c|c|c|}
\hline \multicolumn{2}{|c|}{$(R P \% ; r \%)$} & \multicolumn{2}{|c|}{$\bar{\delta}=0$} & \multicolumn{4}{|c|}{$\delta=-0.25$} \\
\hline$\eta$ & $\frac{w_{1}}{w_{1}+w_{2}}$ & $\varepsilon=0$ & $\varepsilon=0.10$ & $\varepsilon=0$ & $\varepsilon=0.10$ & $\varepsilon=0.20$ & $\varepsilon=0.30$ \\
\hline \multirow[t]{3}{*}{0.5} & 0.5 & $(2.1 ; 3.2)$ & $(2.1 ; 3.5)$ & $(6.3 ; 1.4)$ & $(6.3 ; 1.6)$ & $(6.3 ; 2.4)$ & $(6.3 ; 2.8)$ \\
\hline & 0.9 & $(2.1 ; 3.2)$ & $(0.8 ; 3.9)$ & $(6.3 ; 1.4)$ & $(5.0 ; 2.1)$ & $(3.6 ; 2.9)$ & $(2.3 ; 4.0)$ \\
\hline & 0.1 & $(2.1 ; 3.2)$ & $(3.4 ; 2.7)$ & $(6.3 ; 1.4)$ & $(7.6 ; 0.9)$ & $(9.0 ; 0.6)$ & $(10.3 ; 0.5)$ \\
\hline \multirow[t]{3}{*}{0.9} & 0.5 & $(1.2 ; 1.8)$ & $(1.2 ; 1.9)$ & $(5.4 ; 0.8)$ & $(5.4 ; 0.9)$ & $(5.4 ; 1.0)$ & $(5.4 ; 1.3)$ \\
\hline & 0.9 & $(1.2 ; 1.8)$ & $(-1.2 ; 2.1)$ & $(5.4 ; 0.8)$ & $(4.1 ; 1.1)$ & $(2.7 ; 1.5)$ & $(1.4 ; 1.9)$ \\
\hline & 0.1 & $(1.2 ; 1.8)$ & $(2.5 ; 1.5)$ & $(5.4 ; 0.8)$ & $(6.7 ; 0.5)$ & $(8,1 ; 0,2)$ & $(9.4 ; 0.0)$ \\
\hline \multirow[t]{3}{*}{1.1} & 0.5 & $(0.9 ; 1.5)$ & $(0.9 ; 1.5)$ & $(5.1 ; 0.7)$ & $(5.1 ; 0.6)$ & $(5.1 ; 0.5)$ & $(5.1 ; 0.2)$ \\
\hline & 0.9 & $(0.9 ; 1.5)$ & $(-0.4 ; 1.7)$ & $(5.1 ; 0.7)$ & $(3.8 ; 0.9)$ & $(2.4 ; 1.1)$ & $(1.1 ; 1.3)$ \\
\hline & 0.1 & $(0.9 ; 1.5)$ & $(2.2 ; 1,2)$ & $(5.1 ; 0.7)$ & $(6.4 ; 0.4)$ & $(7.8 ; 0.1)$ & $(9.1 ;-0.3)$ \\
\hline
\end{tabular}

